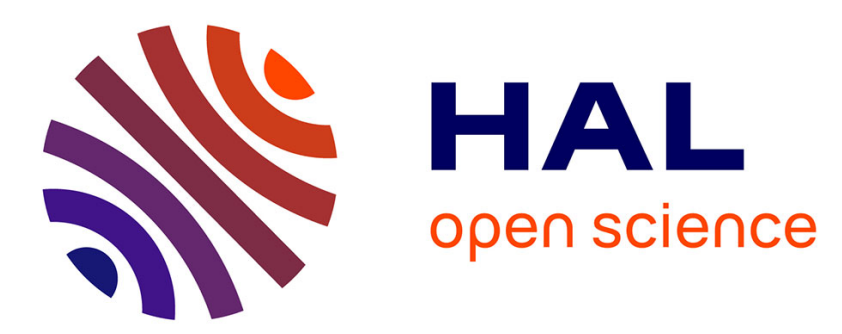

\title{
A method to determine the shear and compressive stress-strain curves from torsion and bending tests of thin wires
}

\author{
Nathanaël Connesson, Gabriel Antherieu, Denis Favier
}

\section{To cite this version:}

Nathanaël Connesson, Gabriel Antherieu, Denis Favier. A method to determine the shear and compressive stress-strain curves from torsion and bending tests of thin wires. Strain, 2017, 53 (3), pp.e12227. hal-02005743

\section{HAL Id: hal-02005743 \\ https://hal.science/hal-02005743}

Submitted on 4 Feb 2019

HAL is a multi-disciplinary open access archive for the deposit and dissemination of scientific research documents, whether they are published or not. The documents may come from teaching and research institutions in France or abroad, or from public or private research centers.
L'archive ouverte pluridisciplinaire HAL, est destinée au dépôt et à la diffusion de documents scientifiques de niveau recherche, publiés ou non, émanant des établissements d'enseignement et de recherche français ou étrangers, des laboratoires publics ou privés. 


\title{
A method to determine the shear and compressive stress-strain curves from torsion and bending tests of thin wires ${ }^{t / 2}$
}

\author{
N. Connesson ${ }^{\mathrm{a}, \mathrm{b}}$, G. Antherieu ${ }^{\mathrm{a}, \mathrm{b}}$, D. Favier ${ }^{\mathrm{a}, \mathrm{b}}$ \\ ${ }^{a}$ Université Grenoble Alpes, TIMC-IMAG, F-38000 Grenoble, France. \\ ${ }^{b}$ CNRS, TIMC-IMAG, F-38000 Grenoble, France.
}

\begin{abstract}
Some materials are provided as wire or other slender geometries and are commonly used as mechanical parts, especially in medical applications. If slender geometries are well adapted to quasi-static tensile tests, such geometries cannot be properly tested under shear and compressive loads. The shear and compressive stress-strain curves nevertheless remain of major interest to engineers and designers. This paper proves and illustrates that material shear and compressive stress-strain curves can efficiently be extracted from torsion and bending tests on thin wires, even in noisy situations. The method is first presented to analyze torsion tests. This method is then developed and adapted to extract the material compressive stress-strain curve from both pure bending and uni-axial tensile tests.
\end{abstract}

Keywords: Beams, Inverse method, Bending, Torsion, Compression,

\section{Introduction}

Experimentally assessing the mechanical behavior of materials is essential since it allows to properly design mechanical parts and to model their behavior through constitutive equations. The results of these experimental tests are often synthesized as a set of stress-strain curves under elementary loads such as shear and uni-axial tensile and compressive loads.

These curves present various shapes over different loading ranges depending on the studied materials. In addition, compressive and tensile load behaviors are not always symmetrical (magnesium [1-3], NiTi Alloys [4], etc), which underlines the necessity of experimental characterizations to assess all these behaviors.

Different mechanical setups have been designed to experimentally identify these sought stressstrain curves. On one hand, classical setups attempt to induce uniform stress state in the tested material (e.g. uni-axial tensile, uni-axial compressive [4] or pure shear tests [5]). These tests are particularly convenient as they straightforwardly provide the desired stress-strain relation. They may

\footnotetext{
This work has been supported by the LABEX CAMI (Ref. ANR-11-LABX-0004).

* Corresponding author: Nathanael Connesson

Email address: nathanael.connesson@univ-grenoble-alpes.fr (N. Connesson)
} 
yet present experimental difficulties: some material display strain localization (Lüders bands [6, 7]) when loaded under tension. From an other point of view, obtaining the compressive behavior of material has always proved challenging as compressive tests are subjected to buckling $[8,9]$. This phenomenon particularly restrains the mechanical characterization of materials provided in the shape of slender specimens such as thin tubes, wires or sheets. For many geometry and materials, reaching a satisfying compression load range is thus difficult. Standard compression methods generally require using specific anti-buckling setups inducing friction during the tests (example and short review in $[2,4])$. However, such setups remain ill adapted to experimentally deal with thin wires or thin walled tubes.

On the other hand, samples can be more easily tested under non uniform stress loadings. In this work, stress loadings such as (1) torsion and (2) pure bending have been studied. The aforementioned stress-strain relations are then contained into the obtained experimental data but are yet not straightforwardly provided. Knowing the applied loading, inverse problems have to be solved to identify parameters of chosen constitutive equations. These equations unfortunately only approximately describe the true material behavior and hence affect the identification of the sought stress-strain relations. Extracting information from complex stress loading thus keeps being an active research field.

(1) Concerning torsion test, literature already documents different methods to extract non linear shear stress-strain relationships from torsion test in the large shear strain range $[10,11]$ for circular cross section specimens. In particular, Yang et al. [11] proposes an efficient method to identify the shear stress-strain curves from torsion tests. These calculations are based on Fourier analysis and are equivalent to the use of a low-pass filter of cut-off pulsation $w_{c}$ to separate high frequency noise from the experimental data $[10,12]$. In this work, it has been chosen to use a chosen function basis to describe the sought non-linear shear stress-strain curves in torsion. A global mean-square approach is used to perform this identification while filtering out the experimental noise. The identified shear stress-strain curves and noise sensitivity of these two methods will be shortly compared in section 3.1 .

(2) Concerning pure bending tests, experimental data contain information about the tensile and compressive material behavior and associated asymmetry, which would be of interest to extract. Yet, to the author knowledge, no attempt has yet been published to directly extract stress-strain relationship from such data. In fact, researches about pure bending behavior are still undergoing, but usually try to predict the bending behavior assuming models (elasto-plastic strain-hardening model [13, 14], non-linear models tacking into account loading-unloading cycles [15, 16], taking into account material inhomogeneity [17], predicting ultimate pure bending [18], etc.). Fewer works 
propose method to extract information from bending test or are interested in information such as the hardening parameters during cyclic loading [19-21]. These predictions yet usually assume specific material models and tension-compression symmetrical behavior which is not true for all materials. Direct extraction of stress-strain curves has thus not been published.

This gap in literature is probably due to (i) the lack of experimental mean to reach large curvature ranges and (ii) to the fact that extracting the elementary stress-strain curves from pure bending data is highly challenging: the extraction robustness depends on the input data quality, the presence of bending experimental noise/bias, the knowledge of specimen section geometry and variations during the test, the curvature identification, etc. Obstacle (i) has been progressively overcame thanks to the recent development of new experimental bending setups (thin plates [22, 23], few millimeter diameter rods [24, 25], cylindrical shells [9]). More recently, the setup developed in [26] provided an efficient experimental system to obtain pure bending measurements on thin wires or tubes over large curvature ranges (curvature radii from infinite to about $2.5 \mathrm{~mm}$ for wires of diameter less than $1 \mathrm{~mm}$ ). The obstacle (ii) is tackled in the present paper; solutions to the many associated challenges are proposed and evaluated.

This paper thus proposes an original method to extract material elementary stress-strain relations from either torsion (section 2.1) or pure bending tests (section 2.2). This study has been restricted to a thorough analyze of the proposed method performances on simulated experiments to evaluate its noise robustness and sensibility to the various parameters described in obstacle (ii) (section 3). Application of this method to analyze real experimental data will be published in further work so as not to overly lengthen this paper.

\section{Method}

From a general point of view, during torsion or pure bending of a wire, the specimen circular cross-section $S$ is loaded by the different components of the tensor stress field $\underline{\underline{\sigma}}_{(\mathrm{M})}$ (Fig. 1), where $\underline{\underline{\boldsymbol{\sigma}}}_{(\mathrm{M})}$ is the Cauchy stress tensor at any point $M$ of the cross-section $S$. The overall resulting force $\vec{R}$ and moment $\overrightarrow{M_{O}}$ in the cross-section $S$ can be simply written:

$$
\begin{gathered}
\vec{R}=\iint_{S} \underline{\underline{\boldsymbol{\sigma}}}_{(\mathrm{M})} \overrightarrow{u_{z}} d S \\
\overrightarrow{M_{O}}=\iint_{S} \overrightarrow{O M} \wedge\left(\underline{\underline{\boldsymbol{\sigma}}}_{(\mathrm{M})} \overrightarrow{u_{z}}\right) d S
\end{gathered}
$$

where $\overrightarrow{u_{z}}$ is the unit vector orthogonal to the cross-section $S$, and $d S$ is an elementary surface area element. The point $O$ is the point of section $S$ where the moment $\overrightarrow{M_{O}}$ is estimated. 
In this paper, overall resulting force $\vec{R}$ and moment $\overrightarrow{M_{O}}$ have been simulated either during torsion (section 3.1) or pure bending tests (section 3.2). The aim of this work is to extract from these tests the material local shear and compressive stress-strain behaviour contained into the Cauchy stress tensor $\underline{\underline{\boldsymbol{\sigma}}}_{(\mathrm{M})}$.

\subsection{Torsion test}

\subsubsection{Method overview}

Torsion is a loading configuration where (i) a specimen (here a wire) is twisted along its principal axis $\mathcal{A}$ by a torque $\overrightarrow{M_{O}^{\text {tors }}}$ (Fig. 2) and (ii) the overall resulting force $\vec{R}$ is zero. In this paper, notations referring to torsion loading conditions has been labeled as $X^{\text {tors }}$. In torsion around $\overrightarrow{u_{z}}$ (Fig. 2), for circular cross-section specimens (tubes or rods), the local Cauchy stress tensor can be expressed in the frame $\left(\overrightarrow{u_{r}}, \overrightarrow{u_{\theta}}, \vec{z}\right)$ as:

$$
\underline{\underline{\boldsymbol{\sigma}}}_{(\mathrm{M})}=\left[\begin{array}{ccc}
0 & 0 & 0 \\
0 & 0 & \tau(\gamma) \\
0 & \tau(\gamma) & 0
\end{array}\right]_{\left(M, \overrightarrow{u_{r}}, \overrightarrow{u_{\theta}}, \vec{z}\right)}
$$

where $\tau$ is the local shear stress and $\gamma$ the local shear strain. Assuming a planar cross-section remains planar during the torsion test and that a radius line remains a line [27, 28], the local shear strain $\gamma$ can be written [28]:

$$
\gamma=\frac{\theta}{L} r=\alpha r
$$

where $\theta$ is the relative rotation between the specimen tips, $L$ is the specimen length and $r$ is the distance between the wire center and any point $M$ of the cross-section $S$ (Fig. 2).The angle $\alpha$ is the relative rotation per specimen length. Equations 1 and 2 can thus be simplified into:

$$
\begin{gathered}
\overrightarrow{R^{\text {tors }}}=\iint_{S} \tau(\gamma) \overrightarrow{u_{\theta}} d S=\overrightarrow{0} \\
\overrightarrow{M_{O}^{\text {tors }}}=\iint_{S} r \overrightarrow{u_{r}} \wedge \tau(\gamma) \overrightarrow{u_{\theta}} d S=\left(\iint_{S} r \tau(\gamma) d S\right) \overrightarrow{u_{z}}
\end{gathered}
$$

During torsion experiments, a set of $P$ experimental torque values with respect to the relative rotation $\left\{M_{O}^{\text {tors }}\left(\alpha_{p}\right)\right\}, p \in[1 ; P]$ is provided. The aim of this method is to identify the shear stress function $\tau(\gamma)$ from these experimental data.

As an overview, the true shear stress function $\tau(\gamma)$ has been approximated by an analytical shear stress function $f^{\text {shear }}(\gamma)$. This sought function $f^{\text {shear }}$ is directly related to its associated analytical

torque function $M_{O}^{\text {tors approx }}$ (Eq. 6). The function $f^{\text {shear }}$ and torque function $M_{O}^{\text {tors approx }}$ are 
thus sought so as to minimize the function $\Phi^{\text {tors }}$ defined in the least mean square sense as:

$$
\Phi^{\text {tors }}=\sum_{p=1}^{P}\left(M_{O}^{\text {tors }}\left(\alpha_{p}\right)-M_{O}^{\text {tors approx }}\left(\alpha_{p}\right)\right)^{2}
$$

\subsubsection{Method details}

More specifically, the shear stress function $f^{\text {shear }}$ has been written as a linear combination of $K$ elementary functions $S_{k}, k \in[1 ; K]$ (e.g. polynomial, spline,etc.) so that:

$$
f^{\text {shear }}(\gamma)=\sum_{k=1}^{K} q_{k} S_{k}(\gamma)=\left\langle S_{1}(\gamma) \cdots S_{K}(\gamma)\right\rangle\left\{\begin{array}{c}
q_{1} \\
\vdots \\
q_{K}
\end{array}\right\}
$$

where $q_{k}$ are the $K$ real coefficients to be identified. These $K$ unknown coefficients $q_{k}$ compose the column matrix $\left\{q_{k}\right\}$; the $K$ elementary functions $S_{k}$ compose the function row matrix $\left\langle S_{1}(\gamma) \cdots S_{K}(\gamma)\right\rangle$. Combining equations 4, 6 and 8, the searched torsion moment $M_{O}^{\text {tors approx }}$ thus naturally writes:

$$
\begin{aligned}
M_{O}^{\text {tors approx }}\left(\alpha_{p}\right) & =\sum_{k=1}^{K}\left(q_{k} \iint_{S} r S_{k}(\gamma) d S\right) \\
& =\sum_{k=1}^{K} q_{k} U_{k}\left(\alpha_{p}\right)=\left\langle U_{1}\left(\alpha_{p}\right) \cdots U_{K}\left(\alpha_{p}\right)\right\rangle\left\{\begin{array}{c}
q_{1} \\
\vdots \\
q_{K}
\end{array}\right\}
\end{aligned}
$$

where the $K$ functions $U_{k}\left(\alpha_{p}\right), k \in[1 ; K]$ are defined as:

$$
U_{k}\left(\alpha_{p}\right)=\iint_{S} r S_{k}(\gamma) d S \quad \text { with } \quad \gamma=\alpha_{p} r
$$

Using Eq. 9, the function $\phi^{\text {tors }}$ (Eq. 7) to be minimized thus eventually writes:

$$
\Phi^{\text {tors }}\left(q_{k}\right)=\sum_{p=1}^{P}\left(M_{O}^{\text {tors }}\left(\alpha_{p}\right)-\sum_{k=1}^{K} q_{k} U_{k}\left(\alpha_{p}\right)\right)^{2}
$$

An additional constraint can also be written as the shear strain is zero when the specimen is not loaded:

$$
f^{\text {shear }}(\gamma=0)=\sum_{k=1}^{K} q_{k} S_{k}(\gamma=0)=\left\langle S_{k}(\gamma=0)\right\rangle\left\{q_{k}\right\}=0
$$

where $\left\langle S_{k}(\gamma=0)\right\rangle$ is a $K$ element row matrix. 
Identifying the mechanical behaviour under shear load $f^{\text {shear }}$ thus consists in solving the corresponding problem $\mathscr{P}^{\text {tors }}$ by finding the unknown column matrix $\left\{q_{k}\right\}$ that minimizes function $\Phi^{\text {tors }}$ (Eq. 12) while respecting the constraint defined by Eq. 13:

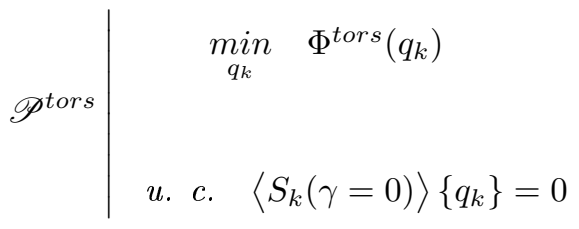

The corresponding Lagrangian $\mathcal{L}_{\text {tors }}$ of the problem $\mathscr{P}^{\text {tors }}$ writes [29]:

$$
\mathcal{L}_{\text {tors }}=\Phi^{\text {tors }}\left(q_{k}\right)-\lambda\left(\sum_{k=1}^{K} q_{k} S_{k}(\gamma=0)-0\right)
$$

where $\lambda$ is the Lagrange multiplier related to the constraint.

From a practical point of view, the unknown column matrix $\left\{q_{k}\right\}$ can simply be obtained by solving the linear system written [29]:

$$
\left[\bar{H}_{\mathcal{L}_{\text {tors }}}\right]\left\{\begin{array}{c}
\left\{q_{k}\right\} \\
\lambda
\end{array}\right\}=\left\{\begin{array}{c}
\left\{M_{O}^{\text {tors }}\left(\alpha_{p}\right)\right\} \\
0
\end{array}\right\}
$$

where $\left[\bar{H}_{\mathcal{L}_{\text {tors }}}\right]$ is the corresponding bordered Hessian $(P+1) \times(P+1)$ matrix written:

$$
\left[\bar{H}_{\mathcal{L}_{\text {tors }}}\right]=\left[\begin{array}{c|c}
{[\mathbf{U}]^{t}[\mathbf{U}]} & \left\{S_{k}(\gamma=0)\right\} \\
\hline\left\langle S_{k}(\gamma=0)\right\rangle & 0
\end{array}\right]
$$

where the notation $X^{t}$ stand for the matrix transposition. The $P \times K$ matrix $[\mathbf{U}]$ is computed by estimating the function row matrix $\left\langle U_{1}\left(\alpha_{p}\right) \cdots U_{K}\left(\alpha_{p}\right)\right\rangle$ (Eqs. 10 and 11) at the different measurement relative angles $\alpha_{p}$; the $P \times K$ matrix $[\mathbf{U}]$ represents the $P$ equations system coupling the approximated torques values $\left\{M_{O}^{\text {tors approx }}\left(\alpha_{p}\right)\right\}$ and column matrix $\left\{q_{k}\right\}$ (Eq. 10):

$$
\left\{M_{O}^{\text {tors approx }}\left(\alpha_{p}\right)\right\}=\left[\begin{array}{cccc}
\left\langle U_{1}\left(\alpha_{1}\right)\right. & U_{2}\left(\alpha_{1}\right) & \cdots & \left.U_{K}\left(\alpha_{1}\right)\right\rangle \\
\left\langle U_{1}\left(\alpha_{2}\right)\right. & U_{2}\left(\alpha_{2}\right) & \cdots & \left.U_{K}\left(\alpha_{2}\right)\right\rangle \\
\vdots & & \ddots & \vdots \\
\left\langle U_{1}\left(\alpha_{P}\right)\right. & \cdots & \cdots & \left.U_{K}\left(\alpha_{P}\right)\right\rangle
\end{array}\right]\left\{\begin{array}{c}
q_{1} \\
\vdots \\
q_{K}
\end{array}\right\}=[\mathbf{U}]\left\{q_{k}\right\}
$$

Once the column matrix $\left\{q_{k}\right\}$ is identified solving equation 16 , the approximated shear stress function $f^{\text {shear }}(\gamma)$ can be computed using equation 8 .

The method analytical identification robustness to experimental noise and bias is shortly studied 
in the next section.

\subsubsection{Error analyze}

Identification errors between the real function $\tau(\gamma)$ and the sought shear stress function $f^{\text {shear }}$ can be due to different factors:

- Elementary functions basis $\left\{S_{k}\right\}(k \in[1 ; K])$ inadequacy: the chosen elementary functions basis $\left\{S_{k}\right\}$ may not be rich enough to properly describe the real material behavior $\tau(\gamma)$. The error induced by this inadequacy can be reduced by increasing the functions basis richness, for example by increasing the number $K$ of elementary functions $S_{k}$. Such an increase will also unfortunately increase the identification sensitivity to noise and a trade-off should be found (see Section 3.1). The functions basis quality can yet be a posteriori analyzed by studying the eventual difference between the experimental and approximated torque $\left(M_{O}^{\text {tors }}\right.$ and $M_{O}^{\text {tors approx }}$, respectively).

- Experimental bias and noise: the experimentally measured torques $\left\{M_{O}^{\text {tors meas }}\left(\alpha_{p}\right)\right\}$ unavoidably contain bias and noise. An experimental measurement can thus be seen as the sum of the real values $\left\{M_{O}^{\text {tors }}\left(\alpha_{p}\right)\right\}$ and the experimental torque error or bias $\left\{\Delta M_{O}^{\text {tors }}\right\}$ so that:

$$
\left\{M_{O}^{\text {tors meas }}\left(\alpha_{p}\right)\right\}=\left\{M_{O}^{\text {tors }}\left(\alpha_{p}\right)\right\}+\left\{\Delta M_{O}^{\text {tors }}\right\}
$$

This noise will induce errors $\left\{\delta q_{k}\right\}$ on the identified values $\left\{q_{k}\right\}$ that will be added to the solution column matrix $\left\{q_{k 0}\right\}$ identified in noiseless conditions. Using Eq. 16 in noisy and noiseless conditions, this error $\left\{\delta q_{k}\right\}$ can be simply estimated by solving the system:

$$
\left[\bar{H}_{\mathcal{L}_{\text {tors }}}\right]\left\{\begin{array}{c}
\left\{\delta q_{k}\right\} \\
\delta \lambda
\end{array}\right\}=\left\{\begin{array}{c}
\left\{\Delta M_{O}^{\text {tors }}\right\} \\
0
\end{array}\right\}
$$

It should be underlined here that this system is independent of the real function $\tau(\gamma)$ and depends only on the elementary chosen functions basis contained into $\left[\bar{H}_{\mathcal{L}_{\text {tors }}}\right]$ and of the experimental torque error or bias $\left\{\Delta M_{O}^{\text {tors }}\right\}$. This equation can thus be used a posteriori to estimate identification error-bars on the results: assuming a bias or noise model allows to estimate influence of the torque experimental noise/bias $\left\{\Delta M_{O}^{\text {tors }}\right\}$ on the identified relation $f^{\text {shear }}$.

- Specimen section geometry: the function $f^{\text {shear }}$ is identified by integrating the elementary functions $\left\{S_{k}\right\}(k \in[1 ; K])$ over the specimen section $S$ (Eq. 11). Computing these functions 
requires thus to assume the specimen section geometry; differences between the experimental and theoretical specimen section will impact the identified function $f$ shear .

The method results and robustness have been analyzed while dealing with numerical torsion results in section 3.1. Moreover, a short comparison of the noise-robustness of this method and the method proposed by [11] has been proposed.

\subsection{Pure bending test}

\subsubsection{Method overview}

The method previously presented to analyze torsion tests has been extended to analyze pure bending tests. For concision sake, only the main steps are presented here as the method has been thoroughly described to deal with torsion test in section 2.1.

Pure bending is a loading configuration where the specimen is a wire bent in one of its plane of

symmetry $P l$ by two opposite, yet equal, couples $\overrightarrow{M_{O}^{b e n d}}= \pm M_{O}^{b e n d} \overrightarrow{u_{x}}$, where $\overrightarrow{u_{x}}$ is orthogonal to the plane of symmetry $P l$ (Fig. 3,a). In this paper, notations referring to pure bending conditions have been labeled $X^{\text {bend }}$. In this configuration:

- the convex specimen part is loaded in tension;

- the concave specimen part is loaded in compression;

- the neutral surface contains fibers that are not subjected to any tensile or compressive stress; the neutral axis is defined by the intersection between the neutral surface and the plane of symmetry $P l$. The specimen neutral surface is located at a distance $y_{0}$ from the median surface and its position may change during the test depending on the applied loading and material tensile/compression asymmetry.

The theoretical stress-strain repartition during such a loading is available in literature for homogeneous linear materials [30] or heterogeneous materials [31, 32]. In this work, the specimen material is supposed homogeneous and no constitutive equations are formulated. Given Bernoulli displacement field hypotheses, the component $\varepsilon_{z z}$ of the Biot strain tensor in the specimen simply writes [30]:

$$
\varepsilon_{z z}=\left(y-y_{0}\right)\left(C-C_{0}\right)
$$

where $C_{0}$ and $C$ are the specimen initial and current curvature, respectively. The parameter $y$ represents the distance between the median surface and any point $M$ of the section $S$. In this work, the different fields and values above and under the neutral surface position $y_{0}$ have been denoted $X^{\text {tens }}$ and $X^{c o m p}$, respectively. 
In case of pure bending around $\overrightarrow{u_{x}}$, the overall resulting force $\overrightarrow{R^{b e n d}}$ is zero and, neglecting the component of stress $\sigma_{y y}[30]$, the corresponding Cauchy stress tensor field $\underline{\underline{\boldsymbol{\sigma}}}_{(\mathrm{M})}$ can be simplified in the frame $\left(\overrightarrow{u_{x}}, \overrightarrow{u_{y}}, \overrightarrow{u_{z}}\right)$ as:

$$
\underline{\underline{\boldsymbol{\sigma}}}_{(\mathrm{M})}=\left[\begin{array}{ccc}
0 & 0 & 0 \\
0 & 0 & 0 \\
0 & 0 & \sigma_{z z}\left(\varepsilon_{z z}\right)
\end{array}\right]_{\left(M, \overrightarrow{u_{x}}, \overrightarrow{u_{y}}, \overrightarrow{u_{z}}\right)}
$$

Using this stress field $\sigma_{z z}$, equations 1 and 2 can be simplified and written as scalar equations while dissociating the tensile and compressive contributions above and under the neutral surface in section $S$ such as:

$$
\begin{aligned}
R^{\text {bend }} & =\iint_{S_{\text {tens }}} \sigma_{z z}^{\text {tens }}\left(\varepsilon_{z z}^{\text {tens }}\right) d S+\iint_{S_{\text {comp }}} \sigma_{z z}^{\text {comp }}\left(\varepsilon_{z z}^{\text {comp }}\right) d S=0 \\
M_{O}^{\text {bend }} & =\iint_{S_{\text {tens }}} y \sigma_{z z}^{t e n s}\left(\varepsilon_{z z}^{t e n s}\right) d S+\iint_{S_{\text {comp }}} y \sigma_{z z}^{\text {comp }}\left(\varepsilon_{z z}^{\text {comp }}\right) d S
\end{aligned}
$$

During pure bending experiments, using the setup presented in [26], a set of $P$ experimental bending moment with respect to curvature $\left\{M_{O}^{\text {bend }}\left(C_{p}\right)\right\}, p \in[1 ; P]$ is provided. The aim of the method is to identify both the function $\sigma_{z z}^{c o m p}\left(\varepsilon_{z z}^{c o m p}\right)$ and the variation of the neutral surface position $\left\{y_{0}\left(C_{p}\right)\right\}$ during the test using equations 23 and 24 .

- In the case in which the specimen material tensile and compressive behavior are symmetrical, a unique stress-strain relation $\sigma_{z z}^{\text {tens }}\left(\varepsilon_{z z}^{\text {tens }}\right)=-\sigma_{z z}^{\text {comp }}\left(-\varepsilon_{z z}^{\text {tens }}\right)$ is sought. Equation 23 can be simplified into:

$$
R^{b e n d}=\iint_{S_{\text {tens }}} \sigma_{z z}^{t e n s}\left(\varepsilon_{z z}^{t e n s}\right) d S-\iint_{S_{\text {comp }}} \sigma_{z z}^{t e n s}\left(-\varepsilon_{z z}^{t e n s}\right) d S=0
$$

For a circular cross-section, equations and 25 and 21 imply that the neutral surface position $y_{0}$ is zero and the surfaces $S_{\text {tens }}$ and $S_{\text {comp }}$ are symmetrical. Equation 24 can thus be simplified into:

$$
M_{O}^{\text {bend }}=2 \iint_{S_{t e n s}} y \sigma_{z z}^{\text {tens }}\left(\varepsilon_{z z}^{\text {tens }}\right) d S
$$

Equations 25 and 26 are very similar to equations 5 and 6 ; adapting the previous method to analyze bending tests is straightforward in such a situation.

- In the case in which the specimen material tensile and compressive behavior are not symmetrical, equations 23 and 24 cannot be simplified as previously. An experimental measurement of the tensile behaviour function $\sigma_{z z}^{\text {tens }}\left(\varepsilon_{z z}^{\text {tens }}\right)$ is thus required.

The method described in section 2.1 has been adapted as an iterative process to identify both 
the function $\sigma_{z z}^{c o m p}\left(\varepsilon_{z z}^{c o m p}\right)$ and the variation of the neutral surface position $\left\{y_{0}\left(C_{p}\right)\right\}$ during the test using equations 23 and 24 . As a summary, during each iteration $c$, the function $\sigma_{z z}^{c o m p}$ is approximated by an analytical compression function $f_{c}^{c o m p}$. This analytical compression function $f_{c}^{c o m p}$ is directly related to an analytical bending function $M_{O, c}^{\text {bend approx }}$ (Eq. 24) and is searched so as to minimize the function $\Phi_{c}^{\text {bend }}$ defined in the least mean square sense as:

$$
\Phi_{c}^{\text {bend }}=\sum_{p=1}^{P}\left(M_{O}^{\text {bend }}\left(C_{p}\right)-M_{O, c}^{\text {bend approx }}\left(C_{p}\right)\right)^{2}
$$

\subsubsection{Method details}

The iterative method has been summarized as a flowchart in Fig 4 and is detailed hereafter:

Initialization $c=0$ :

Initially, the neutral fiber position is assumed to remain at the specimen medium line during the whole test:

$$
\text { If } \mathrm{c}=0, \quad \forall p \in[1, P],\left\{y_{0, c}\left(C_{p}\right)\right\}=\{0\}
$$

Iteration $c(c \geq 1)$ :

As when analyzing the torsion tests, the function $\sigma_{z z}^{c o m p}\left(\varepsilon_{z z}^{c o m p}\right)$ is approximated by an analytical functions $f_{c}^{c o m p}$ at each iteration $c$ :

$$
f_{c}^{c o m p}\left(\varepsilon_{z z}^{c o m p}\right)=\sum_{k=1}^{K} q_{k}^{c} S_{k}\left(\varepsilon_{z z}^{c o m p}\right)=\left\langle S_{1}\left(\varepsilon_{z z}^{c o m p}\right) \cdots S_{K}\left(\varepsilon_{z z}^{c o m p}\right)\right\rangle\left\{\begin{array}{c}
q_{1}^{c} \\
\vdots \\
q_{K}^{c}
\end{array}\right\}
$$

where $f_{c}^{c o m p}$ is the function to be identified during iteration $c$. The functions basis $\left\{S_{k}\right\}, k \in[1 ; K]$ is chosen and defined in a very similar way as for the torsion case (Eq. 8, adapted to bending).

During iteration $c$, the aim is to identify the column matrix $\left\{q_{k}^{c}\right\}$ defining the function $f_{c}^{c o m p}$ as a better approximation than during the previous iteration (function $f_{c-1}^{c o m p}$ ) and the associated neutral fiber position $\left\{y_{0, c}\left(C_{p}\right)\right\}$ during the test.

\section{Step 1-Determination of the coefficients $\left\{q_{k}^{c}\right\}$ and function $f_{c}^{c o m p}$}

Using this basis and equations 24 and 29, the associated analytical bending function $M_{O, c}^{\text {bend approx }}$ simply writes:

$$
M_{O, c}^{\text {bend approx }}\left(C_{p}\right)=\iint_{S_{\text {tens }}} y \sigma_{z z}^{\text {tens }}\left(\varepsilon_{z z}^{\text {tens }}\right) d S+\sum_{k=1}^{K} q_{k}^{c} U_{k}\left(C_{p}\right)
$$

where the functions $\left\{U_{k}\right\}, k \in[1 ; K]$ are defined in a very similar way as to the torsion case (Eq. 11, adapted to bending) using the elementary functions basis $\left\{S_{k}\right\}, k \in[1 ; K]$. 
The integrand $\iint_{S_{t e n s}} y \sigma_{z z}^{\text {tens }}\left(\varepsilon_{z z}^{\text {tens }}\right) d S$ and each function $U_{k}\left(C_{p}\right)$ composing the bending function $M_{O, c}^{\text {bend approx }}$ in Eq. 30 can be numerically computed since the integration sections $S_{\text {tens }}$ and $S_{c o m p}$ have been defined through the neutral surface position $\left\{y_{0, c-1\left(C_{p}\right)}\right\}$ during the previous iteration or initialization. The function $\sigma_{z z}^{\text {tens }}\left(\varepsilon_{z z}^{\text {tens }}\right)$ is known thanks to a previously performed tensile test and the strain field $\varepsilon_{z z}^{t e n s}$ can be estimated in the section $S$ using Eq. 21.

Using Eq. 30, the function $\Phi_{c}^{\text {bend }}$ (Eq. 27) to be minimized during iteration $c$ eventually writes:

$$
\left.\Phi_{c}^{b e n d}\left(q_{k}^{c}\right)=\sum_{p=1}^{P}\left(M_{O}^{b e n d}\left(C_{p}\right)-\iint_{S_{t e n s}} y \sigma_{z z}^{\text {tens }}\left(\varepsilon_{z z}^{\text {tens }}\right)\right) d S-\sum_{k=1}^{K} q_{k}^{c} U_{k}\left(C_{p}\right)\right)^{2}
$$

An additional constraint can then be written as the strain should be zero when the specimen is not loaded:

$$
f^{c o m p}\left(\varepsilon_{z z}^{c o m p}=0\right)=\sum_{k=1}^{K} q_{k}^{c} S_{k}\left(\varepsilon_{z z}^{c o m p}=0\right)=\left\langle S_{k}\left(\varepsilon_{z z}^{c o m p}=0\right)\right\rangle\left\{q_{k}^{c}\right\}=0
$$

The bending problem $\mathscr{P}^{\text {bend }}$ is similar to the torsion problem $\mathscr{P}^{\text {tors }}$. This problem is solved using the same method, i.e. by solving the linear system [29]:

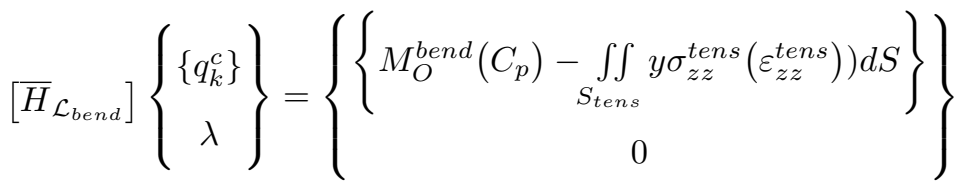

where $\left[H_{\mathcal{L}_{\text {bend }}}\right]$ is defined similarly as for the torsion case (Eq. 17 and 18 using the elementary functions basis defined in the bending case).

Solving this system provides the unknown column matrix $\left\{q_{k}^{c}\right\}$ minimizing the function $\Phi_{c}^{\text {bend }}$ (Eq. 31) and respecting the constraint provided by equation 32 .

The solution column matrix $\left\{q_{k}^{c}\right\}$ can be used to compute the new approximated function $f_{c}^{c o m p}$ and the approximated bending moment $\left\{M_{O, c}^{\text {bend approx }}\left(C_{p}\right)\right\}$ (Eq. 29 and 30, respectively). If the approximated bending moment $\left\{M_{O, c}^{\text {bend approx }}\left(C_{p}\right)\right\}$ is judged too different from the experimental bending moment $\left\{M_{O, c}^{\text {bend }}\left(C_{p}\right)\right\}$, a new spline elementary functions basis $\left\{S_{k}\right\}, k \in[1 ; K]$ can be defined and Step 1 is updated.

\section{Step 2-Determination of neutral fiber position $\left\{y_{0, c}\left(C_{p}\right)\right\}$}

Using the new approximated function $f_{c}^{c o m p}$, equation 23 can be written for each measured curvature $C_{p}$ :

$$
R^{\text {bend }}\left(C_{p}\right)=\iint_{S_{\text {tens }}} \sigma_{z z}^{\text {tens }}\left(\varepsilon_{z z}^{t e n s}\right) d S+\iint_{S_{\text {comp }}} f_{c}^{\text {comp }}\left(\varepsilon_{z z}^{c o m p}\right) d S=0
$$

In this equation and for iteration $c$, only the neutral surface position $\left\{y_{0, c}\left(C_{p}\right)\right\}$ separating the two 
integrated sections $S_{\text {tens }}$ and $S_{\text {comp }}$ is unknown. The position of the neutral surface $\left\{y_{0, c}\left(C_{p}\right)\right\}$ can be estimated by applying a bisection method at any measurement curvature $C_{p}$ so that Eq. 34 is fulfilled.

\section{Step 3-Termination criterion}

Iteration process ends when the difference between two successively identified compressive functions $\left(f_{c-1}^{c o m p}\right.$ and $\left.f_{c}^{c o m p}\right)$ is considered negligible, i.e. when the following termination criterion is verified:

$$
\max \left(\frac{f_{c}^{c o m p}-f_{c-1}^{c o m p}}{f_{c}^{c o m p}}\right) \leq \delta
$$

where $\delta$ is a user chosen value. If the criterion defined by Eq. 35 is verified, $f_{c}^{c o m p}$ and its associated neutral surface position $\left\{y_{0, c}\left(C_{p}\right)\right\}$ are considered to be the sought stress-strain function under compressive load. If the termination criterion is not verified, an additional iteration $c+1$ is performed, starting at Step 1.

\subsubsection{Error analyze}

As for the torsion test, the identification error origins are similar (inadequacy of the chosen functions basis, experimental noise and erroneous specimen section). In particular: the experimentally measured bending moments $\left\{M_{O}^{\text {bend meas }}\left(C_{p}\right)\right\}$ unavoidably contain noise or bias. Using the same notations as presented in Eq. 19 and 20, this noise/bias on the measured torque $\left\{\Delta M_{O}^{\text {bend }}\right\}$ will induce an identification error $\left\{\delta q_{k}\right\}$ added to the solution column matrix $\left\{q_{k 0}\right\}$ identified in noiseless conditions. Using equation 33 in noisy and noiseless conditions, and neglecting the noise influence on the identified final neutral surface position $\left\{y_{0}\left(C_{p}\right)\right\}$, this error $\left\{\delta q_{k}\right\}$ can be simply approximated by solving the system:

$$
\left[\bar{H}_{\left.\mathcal{L}_{\text {bend }}\right]}\right]\left\{\begin{array}{c}
\left\{\delta q_{k}\right\} \\
\delta \lambda
\end{array}\right\}=\left\{\begin{array}{c}
\left\{\Delta M_{O}^{\text {bend }}\right\} \\
0
\end{array}\right\}
$$

As for the torsion case, this system is independent of the real function $\sigma_{z z}^{\text {tens }}\left(\varepsilon_{z z}^{\text {tens }}\right)$ and depends only on the elementary chosen functions basis contained in $\left[\bar{H}_{\mathcal{L}_{\text {bend }}}\right]$ and on the bending moment noise/bias $\left\{\Delta M_{O}^{\text {bend }}\right\}$. By simply assuming a noise model, this equation can be used a posteriori to estimate the identification error-bars.

\section{Validation}

In order to assess the method efficiency and quantify the identification errors, numerical torsion and pure bending simulations have been analyzed. These simulations were performed using stress- 
strain functions used as references; the identified relationships have been thus straightforwardly compared with the reference functions to assess the identification accuracy and robustness.

\subsection{Numerical torsion}

A shear stress-shear strain function $\tau(\gamma)$ has been created so as to simulate a complex mechanical behaviour (Fig. 5a). In particular, this law has been created so as to mimic NiTi alloy mechanical behavior which is of particular interest to the authors [7]. Using this function and a cylindrical specimen $\left(R_{e x t}=0.25 \mathrm{~mm}\right.$, Fig. 2), a torsion test has been simulated (Eq. 6). This simulation provided here $P=180$ values of the moment with respect to the relative rotation $\left\{M_{O}^{\text {tors }}\left(\alpha_{p}\right)\right\}$ for this material behavior (Fig. 5b). These simulated data $\left\{M_{O}^{\text {tors }}\left(\alpha_{p}\right)\right\}$ have been used to analyze the impact of three main parameters on the identification quality:

1. the choice of the elementary functions basis $\left\{S_{k}\right\}(k \in[1 ; K])$,

2. the noise on experimental signal,

3. error on the specimen geometry.

Eventually, the method proposed by Yang in [11] has been applied to the same simulation so as to compare the methods identification performances.

\subsubsection{Functions basis choice}

In this work and for illustration purpose, linear spline functions have been chosen to compose the functions basis $S_{k}$ (results could also have been generated using cubic splines, polynomials or a different kind of functions basis). A first splines basis $\left\{S_{k}\right\}$ composed of $K=5$ linear evenly spaced splines has been first arbitrarily chosen (Fig. 6a, dashed line). The associated normalized functions basis $\left\{U_{k}\right\}$ are also illustrated (Fig. 6b, dashed line, Eq. 11).

The method presented in section 2.1 has been applied with this basis (Fig. 7a and b, dashed line). As a result, the simulated moment-twist results $\left\{M_{O}^{\text {tors }}\left(\alpha_{p}\right)\right\}$ are crudely approximated by the identified function $\left\{M_{O}^{\text {tors approx }}\left(\alpha_{p}\right)\right\}$ obtained with this first splines basis; the observed difference $\left\{M_{O}^{\text {tors approx }}\left(\alpha_{p}\right)-M_{O}^{\text {tors }}\left(\alpha_{p}\right)\right\}$ is unsatisfactory. It is thus obvious that the functions basis $\left\{U_{k}\right\}$ is unadapted to correctly approximate the simulated data $\left\{M_{O}^{\text {tors }}\left(\alpha_{p}\right)\right\}$. As expected, the associated identified shear stress-shear strain function $f^{\text {shear }}$ also crudely approximates function $\tau(\gamma)$ (Fig. $7 \mathrm{~b}$ ). This result underlines the choice importance of the functions basis $\left\{S_{k}\right\}$ and $\left\{U_{k}\right\}$.

This basis choice can be modified until a satisfactory difference between the identified and simulated torsion moments $\left\{M_{O}^{\text {tors approx }}\left(\alpha_{p}\right)-M_{O}^{\text {tors }}\left(\alpha_{p}\right)\right\}$ is obtained. Here, the splines basis support has been refined locally and iteratively until the observed difference $\max \left(\left\{\mid M_{O}^{\text {tors approx }}\left(\alpha_{p}\right)-\right.\right.$ 
$\left.\left.M_{O}^{\text {tors }}\left(\alpha_{p}\right) \mid\right\}\right)$ is less than an arbitrarily chosen threshold of $0.1 \mathrm{Nmm}$; the identified and simulated moments visually overlap (Fig. 7a, continuous line). This second splines basis $\left\{S_{k}\right\}$, composed of $K=8$ linear splines not evenly spaced, and associated functions $\left\{U_{k}\right\}$ resulting of this iterative process are presented in Fig. 6a and b (continuous line). With this new basis, the identified shear stress-shear strain function $f^{\text {shear }}$ is judged satisfactory (Fig. 7b, dashed line).

These results underline (i) the identification method ability, (ii) the functions basis choice decisiveness, (iii) a criterion existence to validate a posteriori the splines basis choice and (iv) the possibility to apply a specific refining method to obtain a satisfactory splines basis $\left\{S_{k}\right\}$.

\subsubsection{Noise impact:}

The identification method robustness to noise has been tested by adding a noise/bias copy $\left\{\Delta M_{0}^{\text {tors }}\right\}$ to the simulated torsion moment $\left\{M_{O}^{\text {tors }}\left(\alpha_{p}\right)\right\}$. This robustness analysis has been performed using the second splines basis previously described (Fig. 6a and b, continuous line). For illustration purpose, the noise has simply been simulated as a normally distributed Gaussian noise, written $\left\{N_{1}\right\}$, of standard deviation $\sigma_{n o i s e}=1 \mathrm{Nmm}$ (i.e. a standard deviation of $6 \%$ of the maximal torsion moment).

The noiseless, noisy input torsion moment and identified moment $\left(\left\{M_{0}^{\text {tors }}\right\},\left\{M_{0}^{\text {tors }}+N_{1}\right\}\right.$ and $\left\{M_{0}^{\text {tors approx }}\right\}$, respectively) are presented Fig. 8a. Due to the noise copy $\left\{N_{1}\right\}$, the identified unknown column matrix $\left\{q_{k} N_{1}\right\}$ and identified moment $\left\{M_{0}^{\text {tors approx }}\right\}$ are different from the unknown column matrix $\left\{q_{k 0}\right\}$ and moment $\left\{M_{0}^{\text {tors approx }}\right\}$ identified in noiseless conditions; the relative error between the results identified in noisy and noiseless condition is here in a range of $\pm 7 \%$ (Fig. $8 \mathrm{a}$, right scale).

The associated identified shear stress function $f_{N_{1}}^{\text {shear }}$ also presents additional error compared to the result obtained in noiseless conditions $f_{0}^{\text {shear }}$ (Fig. 8b). The relative error between the results identified in noisy and noiseless condition is here in a range of $\pm 7 \%$ (Fig. 8b, right scale).

These results are considered excellent considering that the chosen noise standard deviation is $6 \%$ of the maximal torsion moment. It should yet also be mentioned here that the noise impact also depends on the number of measurement $P$ (here, $P=180$ ) contained in the input signal $\left\{M_{0}^{\text {tors }}+N_{1}\right\}$.

The identification error on torsion torque and shear function due to the noise copy $\left\{N_{1}\right\}$ $\left(\left\{\delta M_{0}^{\text {tors }}\right\}=\left\{M_{0 N_{1}}^{\text {tors approx }}\right\}-\left\{M_{0}^{\text {tors approx }}\right\}\right.$ and $\delta f_{N_{1}}^{\text {shear }}=f_{N_{1}}^{\text {shear }}-f_{0}^{\text {shear }}$, respectively $)$ can be predicted by injecting the noise copy $\left\{N_{1}\right\}$ into Eq. 20 to compute the error column matrix $\left\{\delta q_{k N_{1}}\right\}$. This error column matrix is then injected into Eq. 8 and 9 to provide the identification error. Using this method, the errors are perfectly predicted; this prediction has not been presented in a specific figure for concision sake but will be presented while analyzing identification on bending tests. 
While analyzing real experimental data, the specific noise copy/bias $\left\{N_{1}\right\}$ and reference functions are obviously unknown. Nevertheless, simulating different random noise copies $\left\{N_{j}\right\}, j \in[1 ; J]$ provides a family of error functions $\left\{\delta M_{0 N_{j}}^{\text {tors }}\right\}$ and $\left\{\delta f_{N_{j}}^{\text {shear }}\right\}, j \in[1 ; J]$. The Standard-deviations (Std) of these error families have then been computed to simulate the identification error-bar functions ( errorbars $\left._{M}= \pm 2 \operatorname{Std}\left(\delta M_{0}^{\text {tors }} N_{j}\right)\right)$ and $\left(\right.$ errorbars $\left._{f}= \pm 2 \operatorname{Std}\left(\delta f_{N_{j}}^{\text {shear }}\right)\right)$.

These error-bar functions have been plotted on both sides of the reference functions as gray areas in Fig. $8 \mathrm{a}$ and b. The functions $M_{0 N_{1}}^{\text {tors approx }}$ and $f_{N_{1}}^{\text {shear }}$, identified on noisy signal, are contained into the envelopes thus created, emphasizing the method prediction validity: it is thus possible to quantify a posteriori the effect of noise on the identification results by assuming a noise model representing the experimental noise.

Important features to be underlined here are that the error-bar functions errorbars $_{f}$ and $\operatorname{errorbars}_{M}$ (i) are proportional to the Gaussian noise standard-deviation $\sigma_{\text {noise }}$ (Eq. 20), (ii) increase with the loading level (twist angle rate $\alpha$ and shear strain $\gamma$ respectively) and (iii) depend on the chosen splines basis.

This last point can be illustrated by predicting the shear error-bar function errorbar $s_{f}=$ $2 \operatorname{Std}\left(\delta f_{N_{j}}^{\text {shear }}\right)$ for different evenly spaced linear splines basis families and for a standard deviation $\sigma_{\text {noise }}=1 \mathrm{Nmm}$ (Fig. 9). The identification noise sensitivity increases with the number of spline $K$ in the family; the noise filtering ability of a chosen functions basis decreases with the basis richness, which is a classical result. A trade-off should thus be found: the functions basis $\left\{S_{k}\right\}$ should be chosen rich enough so as the experimental torsion moment $M_{O}^{\text {tors }}$ is properly fitted, and simple enough so as to reduce the noise impact on the identification of $f^{\text {shear }}$.

\subsubsection{Difference between experimental and theoretical specimen geometry:}

The identification quality is also dependent on the knowledge of the specimen section geometry $S$. In this work, only wire specimens have been studied. Measuring a diameter $D_{\text {approx }}$ different from the real specimen diameter $D_{\text {true }}$ and using it during the identification process will induce identification errors (Eq. 6). The identification robustness to this geometrical parameter has been studied and compared with the results obtained while identifying shear moduli $G$ on linear materials behaviour.

\section{Linear mechanical behavior:}

For a linear material behavior, estimating the shear function $f^{\text {shear }}$ is equivalent to identify the material shear modulus $G$. Classically, the relation between the shear modulus $G$, the 
torsion torque and the relative rotation $\alpha$ is simply (Eq. 6):

$$
G=\frac{M_{0}^{\text {tors }}}{\alpha} \frac{32}{\pi D^{4}}
$$

The identified shear modulus $G^{\text {approx }}$ using an erroneous measured diameter $D_{\text {approx }}$ would thus simply write:

$$
G^{\text {approx }}=\left(\frac{D_{\text {true }}}{D_{\text {approx }}}\right)^{4} G
$$

This equation indicates that an error as small as $+2.5 \%$ on the diameter measurement $D_{\text {approx }}$ induces an error of $-10 \%$ on the identified shear modulus $G^{\text {approx }}$. Similar conclusion is expected while analyzing complex mechanical behavior.

\section{Complex mechanical behavior:}

For complex material behavior, as previously simulated (Fig. 5a and b, cylindrical specimen, $D_{\text {true }}=0.5 \mathrm{~mm}$ ), a simple relative error equation cannot be derived from Eq. 6 ; the method robustness has been analyzed numerically. The shear function $f$ shear has thus been identified on the simulated data for erroneous diameters $D_{\text {approx }}$ from a range of 90 to $110 \%$ of the real specimen diameter $D_{\text {true }}$. The functions basis to perform this analysis has been chosen identical to the second spline basis previously described (Fig. 6a and b, continuous line).

The identification results are presented in Fig. 10a. As expected, the chosen diameter $D_{\text {approx }}$ affects non linearly the identified shear functions $f^{\text {shear }}$. The overall function shape is yet considered satisfactory. As for the linear case, an overestimated diameter $D_{\text {approx }}$ leads to underestimate the identified shear function $f^{\text {shear }}$ (Fig. 10a and b). Reciprocally, an underestimated diameter $D_{\text {approx }}$ leads to overestimate the identified shear function $f$ shear .

These identification errors are of the same magnitude order than while analyzing linear materials: about $-10 \%$ for an error on the diameter $D_{\text {approx }}$ of $+2.5 \%$.

As a rough estimation, obtaining an error of less than $\pm 1 \%$ on the identified shear function $f$ shear would thus require to estimate the specimen diameter with a precision of $\pm 0.25 \%$. This result underlines the method sensibility to the specimen geometry; its dimensions should be experimentally estimated with great care. Once the other parameters have been chosen, additional error-bars should also be computed by estimating the shear function $f^{\text {shear }}$ boundaries using the diameter experimental measurement tolerance on the specimen section $S$. 


\subsubsection{Method feature comparison with [11]:}

The method proposed by Yang et al in [11] also aims at providing the shear function $f^{\text {shear }}$ from torsion tests. To compare the two methods identification and noise robustness abilities, the previous tests have been performed using Yang's method on the simulated torsion moment $\left\{M_{O}^{\text {tors }}\left(\alpha_{p}\right)\right\}$ : the function $f^{\text {shear }}$ has been identified in noiseless and noisy conditions. Only the main results have been presented for concision sake.

To put it in a nutshell, Yang identify the shear stress-strain curve by expressing equation 6 in the form:

$$
f^{\text {shear }}=\frac{3 M_{0}^{\text {tors }}+\alpha \frac{d M_{0}^{\text {tors }}}{d \alpha}}{2 \pi R_{e x t}^{3}}
$$

where $R_{\text {ext }}$ is the wire external radius. To compute the shear function $f^{\text {shear }}$, equation 39 requires the calculus of the torque derivative versus the relative rotation $\frac{d M_{0}^{\text {tors }}}{d \alpha}$.

This calculus is performed using a trick to create a periodical function using the experimental torque $M_{0}^{\text {tors }}$. This periodical function is then approximated and differentiated using a filtering function basis designed using Fourier analysis theory. This approximation is equivalent to applying to the periodical function a low-pass filter of cut-off pulsation $w_{c}$ [12], removing thus the high frequency harmonics mainly attributed to noise. The approximated function $M_{0}^{\text {tors approx }}$ and associated derivative $\frac{d M_{0}^{\text {tors approx }}}{d \alpha}$ are then reconstructed so as to compute the shear function $f^{\text {shear approx }}$ using equation 39 . The main parameter impacting the identification quality with Yang's method is thus the chosen cut-off pulsation $w_{c}$ which should be adjusted depending on the analyzed signal richness and the noise frequency content. The influence of this parameter is illustrated hereafter in exactly the same layout as previously.

Function basis choice : similarly as previously with the number of splines $K$ in the spline basis, the chosen cut-off pulsation $w_{c}$ affects the approximation basis richness. It is thus obvious that the approximated torque and shear functions $\left\{M_{O}^{\text {tors approx }}\right\}$ and $\left\{f_{0}^{\text {shear approx }}\right\}$ are different from the simulated functions $\left\{M_{O}^{\text {tors }}\right\}$ and $\left\{f^{\text {shear }}\right\}$ even if the identification is performed in noiseless conditions (figure 11a) and b), respectively). These results are comparable with those obtained for the presented method in figure 7 . To allow a fare comparison of the method results, the cut-off pulsation $w_{c}$ has been increased until the observed difference $\max \left(\left\{\left|M_{O}^{\text {tors approx }}\left(\alpha_{p}\right)-M_{O}^{\text {tors }}\left(\alpha_{p}\right)\right|\right\}\right)$ is less than the previously arbitrarily chosen threshold of $0.1 \mathrm{Nmm}$; this criterion has been satisfied here for a cut-off pulsation $w_{c}=0.10 \mathrm{rad} \mathrm{s}^{-1}$.

Noise impact : due to the noise in the data, the functions identified in noisy and noiseless situation are different. Thanks to the superposition theorem applied to equation 39 and to the filtering 
function basis, the error $\left\{\delta f_{N_{1}}^{\text {shear }}\right\}=\left\{f_{N_{1}}^{\text {shear }}\right\}-\left\{f_{0}^{\text {shear }}\right\}$ can easily be written such as:

$$
\left\{\delta f_{N_{1}}^{\text {shear }}\right\}=[F]\left\{\Delta M_{O}^{\text {tors }}\right\}
$$

where $\left\{\Delta M_{O}^{\text {tors }}\right\}$ represents the torque moment noise/bias and matrix $[F]$ depends on the chosen filtering function basis. The matrix $[F]$ thus depends on the cut-off pulsation $w_{c}$ but is independent on the material behavior.

As previously, a Gaussian noise model has been assumed to simulate the torque moment noise $\left\{\Delta M_{O}^{\text {tors }}\right\}$. Different random noise copies $\left\{N_{j}\right\}, j \in[1 ; J]$ of standard deviation $\sigma_{\text {noise }}=$ $1 \mathrm{Nmm}$ (i.e. a standard deviation of $6 \%$ of the maximal torsion moment) are thus computed.

The associated family of error functions $\left\{\delta f_{N_{j}}^{\text {shear }}\right\}, j \in[1 ; J]$ have then been estimated using equation 40. The shear error-bar functions errorbars $f=2 \operatorname{Std}\left(\delta f_{N_{j}}^{\text {shear }}\right)$ can thus be plotted for different cut-off pulsation values (figure 12). The obtained results are similar in both methods (see figure 9): (1) the error-bar on the identified shear function increases with the shear strain $\gamma$ and (2) increasing the cut-off pulsation $w_{c}$, which is equivalent to increasing the richness of the function basis, increases the noise sensitivity.

In particular, the error-bar function obtained for $w_{c}=0.10 \mathrm{rad} \mathrm{s}^{-1}$ should be compared to the spline basis composed of $K=9$ linear splines both chosen so that the difference $\max \left(\left\{\left|M_{O}^{\text {tors approx }}\left(\alpha_{p}\right)-M_{O}^{\text {tors }}\left(\alpha_{p}\right)\right|\right\}\right)$ is smaller that the threshold $0.1 \mathrm{Nmm}$ (figures 9 and 12): the identification noise robustness for the two method is similar.

\subsubsection{Intermediary conclusion:}

These results confirm the method ability to identify the shear stress-shear strain functions from noisy numerical torsion test. The functions basis $\left\{S_{k}\right\}$ should be iteratively refined until the obtained error $\left\{M_{O}^{\text {tors approx }}\left(\alpha_{p}\right)\right\}-\left\{M_{O}^{\text {tors }}\left(\alpha_{p}\right)\right\}$ is small enough. The user should also be aware that refining the functions basis $\left\{S_{k}\right\}$ increases the overall identification noise sensitivity. This noise sensitivity can be estimated a posteriori by estimating the identification errors by assuming an experimental noise/bias model. Special care should also be taken to properly measure the specimen section $S$ dimension; two different identifications should be performed using the section measurement tolerance interval.

\subsection{Numerical pure bending}

The method features and robustness have also been studied in the case of pure bending. Stress-strain functions under tensile and compressive load were created so as to simulate complex mechanical behaviours for which the compressive behavior is stiffer than the tensile behavior 
(Fig. 13a, dashed line). A pure bending simulation has then been performed for wire specimens of radius $R_{e x t}=0.25 \mathrm{~mm}$. The theoretical variations of the reference neutral surface position $y_{0}$ with respect to curvature $C_{p}$ and the associated pure bending moment $M_{0}^{\text {bend }}$ have been computed using equations 23 and 24 . The obtained pure bending moment $\left\{M_{0}^{\text {bend }}\left(C_{p}\right)\right\}$ and associated neutral surface relative position $\frac{y_{0}\left(C_{p}\right)}{R}$ have been plotted in Fig. 13b and c, respectively.

\subsubsection{Functions basis choice}

As for the torsion case, linear splines have been chosen to compose the functions basis $\left\{S_{k}\right\}$. At each iteration $c$, the refining method established in the shear case has been applied to define a splines basis $\left\{S_{k}\right\}$ for which the approximated bending moment results $\left\{M_{O, c}^{\text {bend approx }}\left(C_{p}\right)\right\}$ correctly fit the simulated bending moment function $\left\{M_{O}^{\text {bend }}\left(C_{p}\right)\right\}$; the splines basis $\left\{S_{k}\right\}$ is chosen so that the difference $\left\{M_{O, c}^{\text {bend approx }}\left(C_{p}\right)\right\}-\left\{M_{O}^{\text {bend }}\left(C_{p}\right)\right\}$ is less than an arbitrarily chosen threshold of $0.1 \mathrm{Nmm}$ for each curvature $C_{p}$.

The compressive function $f_{c}^{c o m p}$ identified after convergence and the associated neutral fiber position $\left\{y_{0, c}\left(C_{p}\right)\right\}$ are presented in figure $14 \mathrm{a}$ and $\mathrm{b}$, respectively. The convergence criterion (Eq. 35) has been here verified at the eighth iteration $\left(c=8\right.$ and $\left.\delta=10^{-3}\right)$ and the splines basis is composed of 6 non-evenly spaced linear splines. The final splines basis repartition has been summarized by representing the connection points as square markers directly on the compressive functions $f_{c}^{c o m p}$; the identified functions $f_{c}^{c o m p}$ are piecewise linear due to the linear splines basis choice.

As a result, the identified compressive function $f_{c}^{c o m p}$ almost overlaps the reference functions; the obtained result is judged satisfactory. A smaller error could have been obtained by requiring a smaller error $\left\{M_{O, c}^{\text {bend approx }}\left(C_{p}\right)\right\}-\left\{M_{O}^{\text {bend }}\left(C_{p}\right)\right\}$ while choosing the splines basis $\left\{S_{k}\right\}$.

The neutral surface position $\left\{y_{0, c}\left(C_{p}\right)\right\}$ is also correctly identified. For curvature values lower than $20 \mathrm{~m}^{-1}$, the neutral surface position $\left\{y_{0, c}\left(C_{p}\right)\right\}$ estimation is more sensitive to errors on the identified compression function. The constant value of the neutral fiber position $\left\{y_{0, c}\left(C_{p}\right)\right\}$ for curvatures lower than $20 \mathrm{~m}^{-1}$ is due both to the use of a linear splines basis and to the linear behavior of the material for strain lower than $0.5 \%$.

As a conclusion, these results confirm the method ability to properly identify the compressive function $f^{c o m p}$ and neutral surface position $\left\{y_{0}\left(C_{p}\right)\right\}$ using both the material tensile function $\sigma_{z z}^{\text {tens }}\left(\varepsilon_{z z}^{\text {tens }}\right)$ and the bending moment $M_{0}^{\text {bend }}\left(C_{p}\right)$ as input data. As for the torsion case, the functions basis choice is decisive in the identification quality but a validation of the chosen basis can be performed by verifying that the difference $\left\{M_{O, c}^{\text {bend approx }}\left(C_{p}\right)\right\}-\left\{M_{O}^{\text {bend }}\left(C_{p}\right)\right\}$ is lower than a user-chosen value. 


\subsubsection{Noise impact:}

While analyzing bending, two signals are used as input data: the tensile function $\sigma_{z z}^{\text {tens }}\left(\varepsilon_{z z}^{\text {tens }}\right)$ and the bending moment $\left\{M_{0}^{\text {bend }}\left(C_{p}\right)\right\}$. In this section, it has been chosen to analyze only the impact of the noise on the bending moment $\left\{M_{0}^{\text {bend }}\left(C_{p}\right)\right\}$. For illustration purpose, the noise has been simulated as a normally distributed Gaussian noise of standard deviation $\sigma_{\text {noise }}=0.64 \mathrm{Nmm}$ (i.e. a standard deviation of $8 \%$ of the maximal bending moment in case(1)).

A noise copy $\left\{N_{1}\right\}$ has been added to the input bending moment $\left\{M_{0}^{\text {bend }}\left(C_{p}\right)\right\}$. The noiseless and noisy moments $\left(\left\{M_{0}^{\text {bend }}\right\}\right.$ and $\left\{M_{0}^{\text {bend }}\right\}+\left\{N_{1}\right\}$, respectively) are presented Fig. 15a. The reference bending moment $\left\{M_{0}^{\text {bend }}\left(C_{p}\right)\right\}$ is visually well approximated by the identified bending moment $\left\{M_{0}^{\text {bend approx }}\left(C_{p}\right)\right\}$. As expected, the identified compressive function $f_{N_{1}}^{\text {comp }}$ and neutral surface position $\left\{y_{0 N_{1}}\left(C_{p}\right)\right\}$ are different from the results obtained in noiseless condition $\left(f_{0}^{\text {comp }}\right.$ and $\left\{y_{0}\left(C_{p}\right)\right\}$, respectively, Fig. 15b and c), illustrates the bending moment noise/bias effect during an identification. Considering the noise level applied to the bending moment $\left\{M_{0}^{\text {bend }}\right\}+\left\{N_{1}\right\}$, these results are considered satisfactory.

An approximated model to predict the effect of the specific noise copy $\left\{N_{1}\right\}$ on the identification has been proposed in section 2.2.3. The difference between the compressive function identified in noisy and noiseless condition $\left(f_{N_{1}}^{c o m p}-f_{0}^{c o m p}\right)$ has been compared with the identification analytical error predicted thanks to equations 29 and 36 (Fig. 16). A small difference is observed between the prediction of analytical and numerical error induced by the noise copy $N_{1}$ : this difference is due to the fact that the noise effect on the identified neutral surface position $\left\{y_{0}\left(C_{p}\right)\right\}$ has been neglected to provide equation 36 . The error prediction induced by the noise copy $N_{1}$ on the compressive function $f_{N_{1}}^{c o m p}-f_{0}^{c o m p}$ is yet considered excellent.

While analyzing real experimental data, the specific noise copy $\left\{N_{1}\right\}$ and reference functions are obviously unknown. Nevertheless, simulating different random noise copies $\left\{N_{j}\right\}, j \in[1 ; J]$ and using equations 36 and 29 provides a family of error functions $\left\{\delta M_{0}^{b e n d}\right\}$ and $\left\{\delta f_{N_{j}}^{c o m p}\right\}, j \in[1 ; J]$. The standard-deviations of these error families have then been computed to estimate the identification error-bar functions ( $\left.\operatorname{errorbar}_{M}= \pm 2 \operatorname{Std}\left(\delta M_{0}^{\text {bend }}\right)\right)$ and $\left(\operatorname{errorbars}_{f}= \pm 2 \operatorname{Std}\left(\delta f_{N_{j}}^{\text {comp }}\right)\right)$. The error-bar functions have been plotted on both sides of the reference function as gray areas (Fig. 15a and b): the identified compressive function $f_{N_{1}}^{c o m p}$ is contained into the computed errorbars $\left(\right.$ errorbars $\left.= \pm 2 \mathbf{S t d}\left(\delta f_{N_{j}}^{c o m p}\right)\right)$. This result thus validates the proposed method to a posteriori compute error-bars during bending analysis using equation 36 .

Eventually, exactly as for the torsion case, the error-bar functions (i) are proportional to the Gaussian noise standard-deviation $\sigma_{\text {noise }}$ (Eq. 36), (ii) increase with the compressive strain $\epsilon_{z z}^{c o m p}$ and (iii) depend on the chosen splines basis $\left\{S_{k}\right\}$ (not presented here for concision sake). 
These results confirm the ability of the method to identify the neutral surface position $\left\{y_{0}\left(C_{p}\right)\right\}$ and the stress-strain function under compressive load $f^{c o m p}$ using uni-axial tensile and noisy pure bending test results.

\subsubsection{Difference between experimental and theoretical specimen geometry:}

As for torsion tests, the identification method robustness is dependent on the knowledge of the specimen section geometry $S$. In this work, only cylindrical specimen sections have been studied. Using a diameter $D_{\text {approx }}$ different from the real specimen diameter $D_{\text {true }}$ during the identification process will induce identification errors (Eq. 23 and 24). The identification robustness to this geometrical parameter has been be compared while analyzing linear or more complex mechanical behavior.

\section{Linear mechanical behavior:}

For a linear material behavior symmetrical in tension and compression, estimating the compression function $f^{c o m p}$ is equivalent to identify the material Young modulus E. Classically, the relation between the Young modulus $E$, the bending moment $M_{0}^{\text {bend }}$ and the curvature $C$ simply writes (Eq. 24):

$$
E=\frac{M_{0}^{\text {bend }}}{C} \frac{64}{\pi D^{4}}
$$

The identified Young modulus $E^{a p p r o x}$ is thus similar in bending and torsion cases and simply write:

$$
E^{\text {approx }}=\left(\frac{D_{\text {true }}}{D_{\text {approx }}}\right)^{4} E
$$

The conclusions are similar as for the shear modulus $G$ identification.

\section{Complex mechanical behavior:}

For a complex material behavior, a simple relative error equation cannot be derived from Eq. 24; the method robustness has been analyzed numerically by identifying the compressive function $f^{c o m p}$ on the simulated data for erroneous diameters $D_{\text {approx }}$ from a range of 95 to $105 \%$ of the real specimen diameter $D_{\text {true }}$.

The identified compressive function $f^{\text {comp }}$ and neutral surface relative position $\left\{y_{0}\left(C_{p}\right)\right\}$ have been presented in Fig. 17a and b, respectively. As expected, the section $D_{\text {approx }}$ strongly affects both the identified functions $f^{c o m p}$ and neutral surface relative position $\left\{y_{0}\left(C_{p}\right)\right\}$; a diameter $D_{\text {approx }}$ smaller than the real specimen diameter $D_{\text {true }}$ induces a general increase of the identified function $f^{\text {comp }}$ to obtain the required bending moment $\left\{M_{0}^{\text {bend }}\left(C_{p}\right)\right\}$. The error on the measured diameter $D_{\text {approx }}$ yet induces greater error than in the torsion case: an error as small as $+1 \%$ on the diameter $D_{\text {approx }}$ induces an error of about $-2 \%$ on the 
identified neutral position and of about $-10 \%$ on the identified functions $f^{c o m p}$. This error is greater than the $4 \%$ error predicted in the identification of the Young modulus $E$ for linear mechanical behavior (Eq. 42). In fact, in equation 41, the Young modulus is identified assuming symmetrical compressive and tensile behavior, whereas in the proposed method, the tensile function $f^{t e n s}$ is defined through a tensile test: only the compressive function $f^{\text {comp }}$ is identified to obtain the required bending moment $\left\{M_{0}^{\text {bend }}\right\}$. This phenomenon explains the greater error obtained with the proposed method compared to the results predicted by equation 42.

This result underlines the method identification sensibility to the specimen section geometry $S$; its dimensions should be experimentally estimated with great care. Once the other parameters have been chosen, additional error-bars should be computed by estimating the compressive function $f^{\text {comp }}$ boundary using the experimental measurement tolerance on the specimen section $S$.

\section{Conclusion}

An original method to identify the shear-stress shear-strain function from experimental torsion tests has been thoroughly presented. This method has been extended to identify the stress-strain function under compressive load using experimental uni-axial tensile and pure bending tests as input data. Using numerically simulated results of torsion and bending experiments, the method has been proved able to identify the afore-mentioned stress-strain functions in various configurations. The identification robustness analyze proved to be equivalent to the method proposed in [11] when dealing with torsion tests. Moreover, the proposed method identification quality is mainly dependent on three parameters:

1. The functions basis $\left\{S_{k}\right\}$ which is chosen to described the sought function. A criterion is provided to a posterori validate the functions basis choice. A refining method can be applied to obtain a satisfactory functions basis $\left\{S_{k}\right\}$.

2. The experimental noise. A method is provided to a posterori estimate the identification errorbars induced by experimental noise using a noise model. The method proves to be very robust to noise.

3. The knowledge of the specimen section geometry. This parameter should be experimentally determined with great care. Additional error-bars should be computed by estimating the sought functions using the minimum and maximum of the specimen section geometry as input parameters.

In future work, the presented method will be applied to experimental data and to analyze shear and compressive behavior of NiTi materials. 
[1] X. Y. Lou, M. Li, R. K. Boger, S. R. Agnew, and R. H. Wagoner, "Hardening evolution of AZ31B Mg sheet," International Journal of Plasticity, vol. 23, no. 1, pp. 44 - 86, 2007.

[2] P. Zhou, E. Beeh, and H. E. Friedrich, "A Novel Testing Method for uni-axial Compression of Thin-Sheet Magnesium Alloys," Experimental Mechanics, pp. 1-7, 2015.

[3] J. Jain, W. J. Poole, C. W. Sinclair, and M. A. Gharghouri, "Reducing the tension-compression yield asymmetry in a Mg-8Al-0.5Zn alloy via precipitation," Scripta Materialia, vol. 62, no. 5, pp. $301-304,2010$.

[4] L. Orgéas and D. Favier, "Stress-induced martensitic transformation of a NiTi alloy in isothermal shear, tension and compression," Acta Materialia, vol. 46, no. 15, pp. 5579 - 5591, 1998.

[5] P. Manach and D. Favier, "Shear and tensile thermomechanical behavior of near equiatomic niti alloy," Materials Science and Engineering: A, vol. 222, no. 1, pp. 45-57, 1997.

[6] H. Louche and A. Chrysochoos, "Thermal and dissipative effects accompanying Lüders band propagation," Materials Science and Engineering: A, vol. 307, no. 1-2, pp. 15 - 22, 2001.

[7] D. Favier, H. Louche, P. Schlosser, L. Orgéas, P. Vacher, and L. Debove, "Homogeneous and heterogeneous deformation mechanisms in an austenitic polycrystalline $\mathrm{Ti}-50.8$ at.\% $\mathrm{Ni}$ thin tube under tension. Investigation via temperature and strain fields measurements," Acta Materialia, vol. 55, no. 16, pp. 5310 - 5322, 2007.

[8] S. P. Timoshenko and J. M. Gere, Theory of Elastic Stability. Dover Publications, 2012.

[9] S. Kyriakides and G. T. Ju, "Bifurcation and localization instabilities in cylindrical shells under bending-I. Experiments," International Journal of Solids and Structures, vol. 29, no. 9, pp. $1117-1142,1992$.

[10] H. Wu, Z. Xu, and P. Wang, "The Shear Stress-Strain Curve Determination from Torsion Test in the Large Strain Range," Journal of Testing and Evaluation, vol. 20, no. 6, pp. 396-402, 1992.

[11] Z. X. Yang, X. S. Li, and J. Yang, "Interpretation of torsional shear results for nonlinear stress-strain relationship," International Journal for Numerical and Analytical Methods in Geomechanics, vol. 32, no. 10, pp. 1247-1266, 2008.

[12] X. Li, J. Yang, and H. Liu, "Differentiation of Noisy Experimental Data for Interpretation of Nonlinear Stress-Strain Behavior," Journal of Engineering Mechanics, vol. 124, no. 7, pp. 705$712,1998$. 
[13] M. Sitar, F. Kosel, and M. Brojan, "Numerical and experimental analysis of elastic-plastic pure bending and springback of beams of asymmetric cross-sections," International Journal of Mechanical Sciences, vol. 90, pp. $77-88,2015$.

[14] S. Baragetti, "A Theoretical Study on Nonlinear Bending of Wires," Meccanica, vol. 41, no. 4, pp. 443-458, 2006.

[15] L. Sun and R. Wagoner, "Complex unloading behavior: Nature of the deformation and its consistent constitutive representation," International Journal of Plasticity, vol. 27, no. 7, pp. 1126 $-1144,2011$.

[16] L. Orgeas and D. Favier, "Application of the Beam Theory to Model the Pseudoelastic and Ferroelastic Bending of SMA Beams," J. Phys. IV France, vol. 05, no. C2, pp. C2-519-C2-524, 1995.

[17] G. Nie and Z. Zhong, "Closed-form solutions for elastoplastic pure bending of a curved beam with material inhomogeneity," Acta Mechanica Solida Sinica, vol. 27, no. 1, pp. 54 -64, 2014.

[18] M. A. Shahin and M. F. Elchalakani, "A new model based on evolutionary computing for predicting ultimate pure bending of steel circular tubes," Journal of Constructional Steel Research, vol. 94, pp. $84-90,2014$.

[19] P.-A. Eggertsen and K. Mattiasson, "An efficient inverse approach for material hardening parameter identification from a three-point bending test," Engineering with Computers, vol. 26, no. 2, pp. 159-170, 2009.

[20] F. Yoshida, M. Urabe, and V. Toropov, "Identification of material parameters in constitutive model for sheet metals from cyclic bending tests," International Journal of Mechanical Sciences, vol. 40, no. 2-3, pp. $237-249,1998$.

[21] M. Brunet, F. Morestin, and S. Godereaux, "Nonlinear Kinematic Hardening Identification for Anisotropic Sheet Metals With Bending-Unbending Tests," Journal of Engineering Materials and Technology, vol. 123, no. 4, pp. 378-383, 2000.

[22] G. Arnold, S. Calloch, D. Dureisseix, and R. Billardon, "A pure bending machine to identify the mechanical behaviour of thin sheets," in 6th. International ESAFORM Conference on Material Forming, (France), pp. 1-4, Apr. 2003.

[23] J. M. Muñoz-Guijosa, V. Rodríguez de la Cruz, D. Fernández Caballero, A. Díaz Lantada, and J. Echávarri Otero, "Simple Testing System for Pure Bending Tests with Large Deflections," Experimental Mechanics, vol. 52, no. 7, pp. 679-692, 2011. 
[24] B. Reedlunn, C. B. Churchill, E. E. Nelson, J. A. Shaw, and S. H. Daly, "Tension, compression, and bending of superelastic shape memory alloy tubes," Journal of the Mechanics and Physics of Solids, vol. 63, pp. 506 - 537, 2014.

[25] J. P. M. Hoefnagels, C. A. Buizer, and M. G. D. Geers, Experimental and Applied Mechanics, Volume 6: Proceedings of the 2011 Annual Conference on Experimental and Applied Mechanics, ch. A miniaturized contactless pure-bending device for in-situ SEM failure analysis, pp. 587596. New York, NY: Springer New York, 2011.

[26] G. Antherieu, N. Connesson, D. Favier, P. Mozer, and Y. Payan, "Principle and Experimental Validation of a new Apparatus Allowing Large Deformation in Pure Bending: Application to thin Wire," Experimental Mechanics, pp. 1-8, 2015.

[27] B. Wack, "The torsion of a tube (or a rod): General cylindrical kinematics and some axial deformation and ratchet measurements," Acta Mechanica, vol. 80, no. 1, pp. 39-59, 1989.

[28] F. D. Murnaghan, "Finite deformations of an elastic solid," American Journal of Mathematics, vol. 59, no. 2, pp. 235-260, 1937.

[29] I. S. Sokolnikoff and R. M. Redheffer, Mathematics of Physics and Modern Engineering. McGraw-Hill, 2nd revised edition ed., 1966.

[30] S. P. Timoshenko and J. N. Goodier, Theory of Elasticity. McGraw-Hill book Company, 2nd edition ed., 1951.

[31] J. Dryden, "Bending of inhomogeneous curved bars," International Journal of Solids and Structures, vol. 44, no. 11-12, pp. 4158 - 4166, 2007.

[32] S. G. Lekhnitskii, "On the bending of a plane inhomogenbous curved beam," Journal of Applied Mathematics and Mechanics, vol. 43, no. 1, pp. 198 - 200, 1979. 


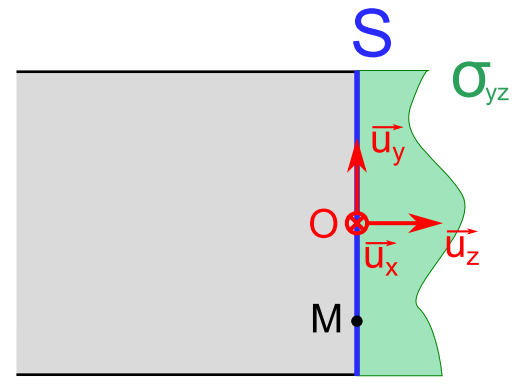

Figure 1: General notation schematic: specimen section $S$ and associated stress field $\sigma_{y z}$.

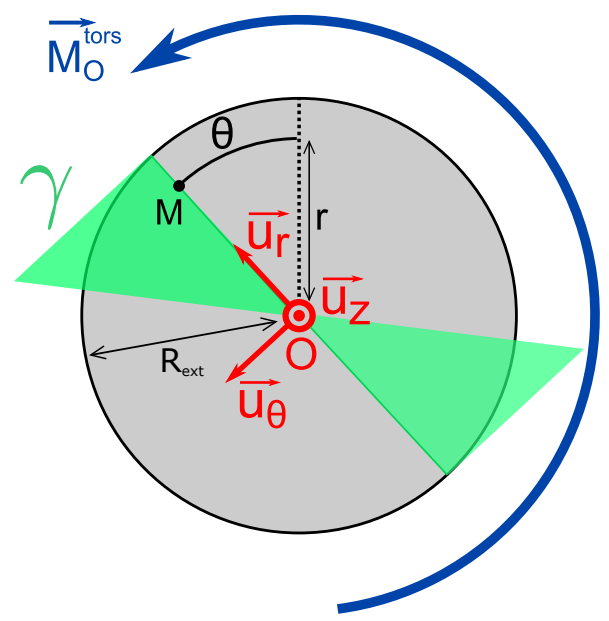

Figure 2: Torsion schematic: shear strain field repartition $\gamma$.
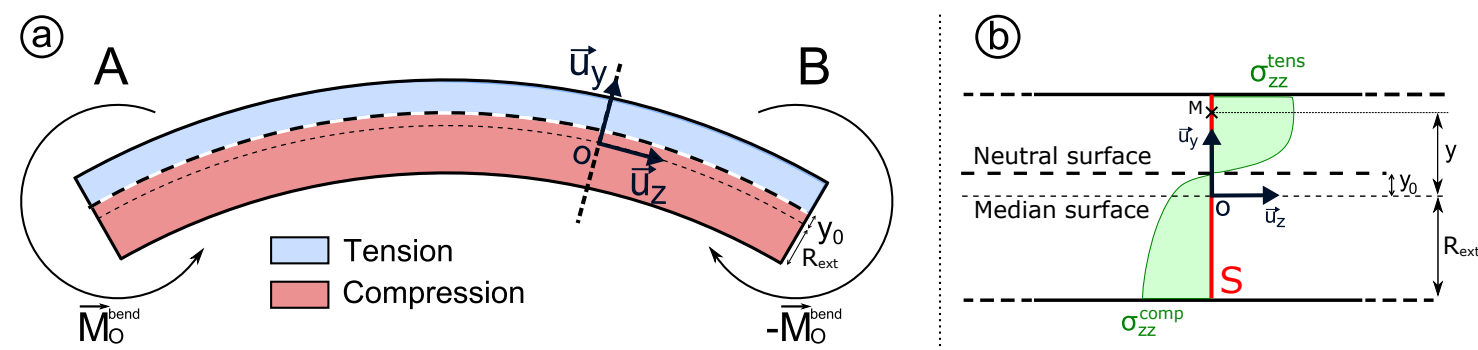

Figure 3: Pure bending schematic: a) Loading configuration in the bending plane $P l$, b) Stress field repartition $\sigma_{z z}$. 


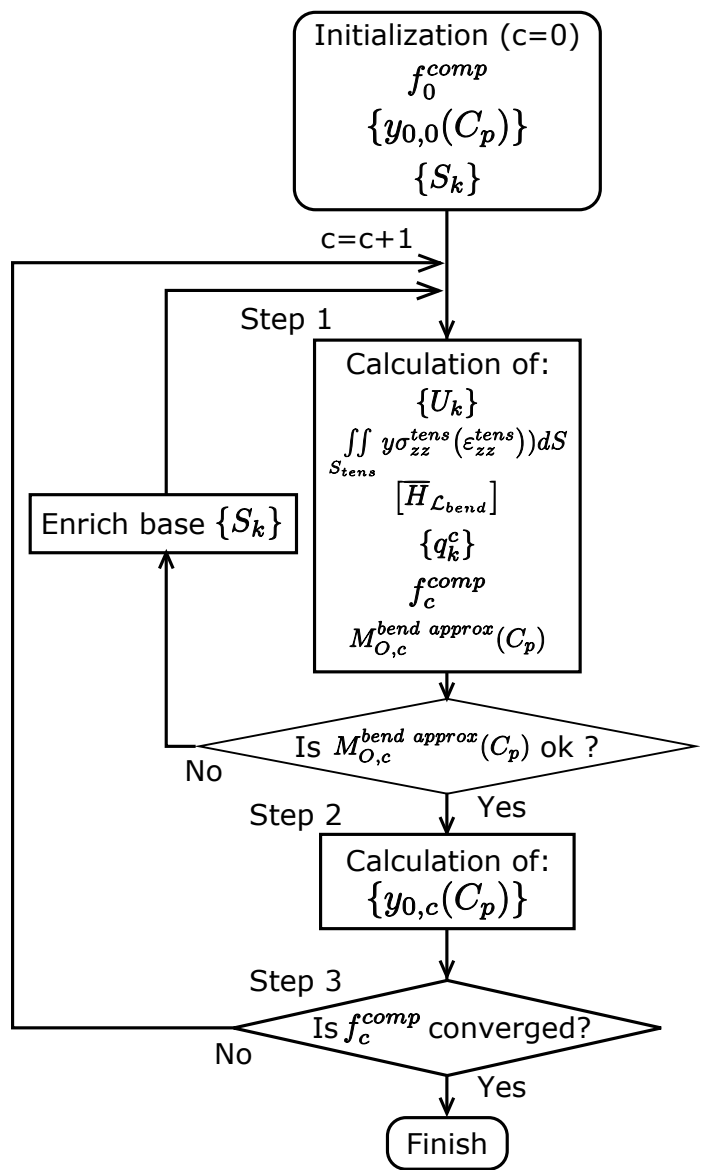

Figure 4: Flowchart used to identify the compressive function $f^{\text {comp }}$. 

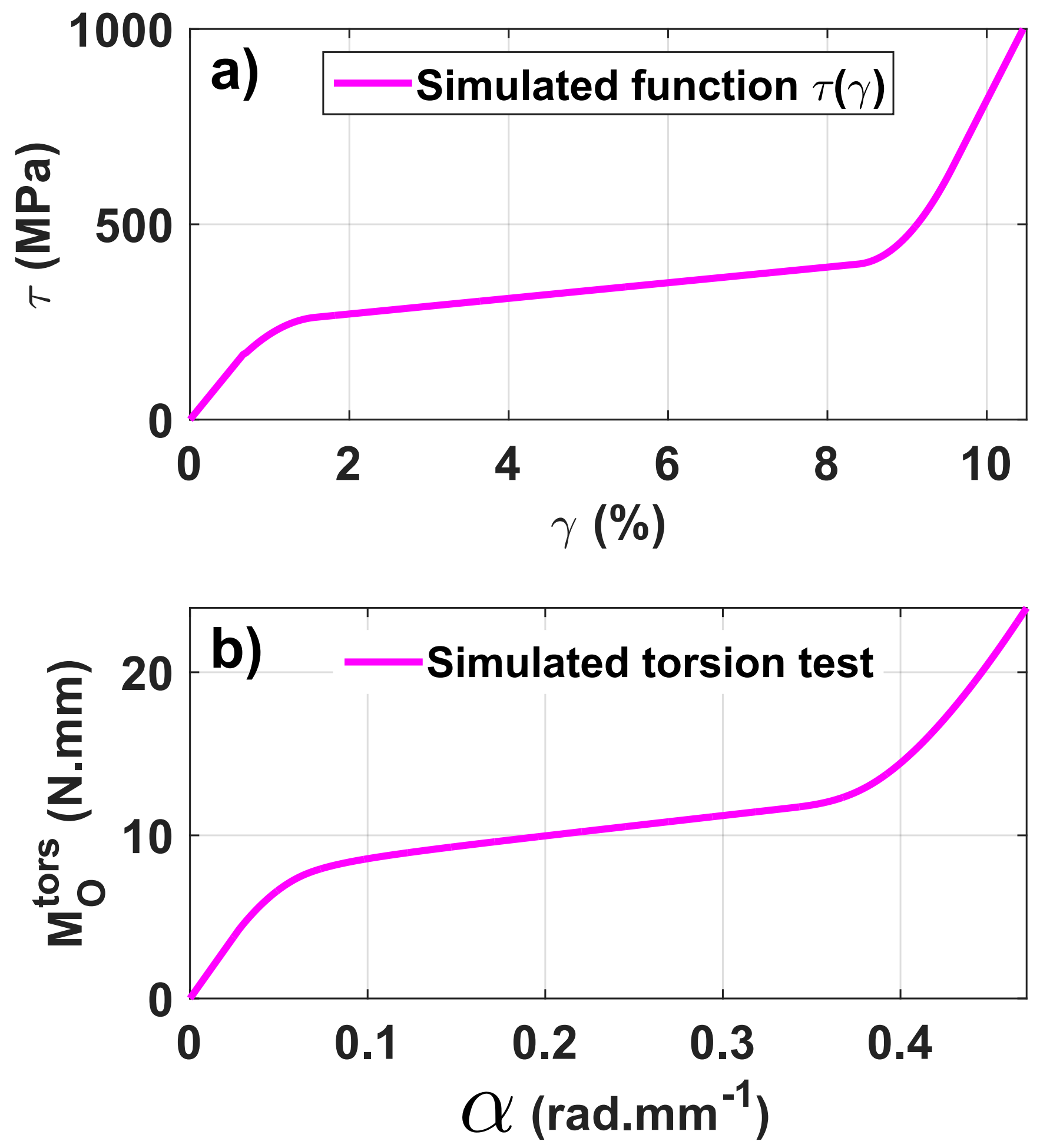

Figure 5: Simulated data: (a) Chosen stress-strain function under shear load, (b) Corresponding simulated torsion test $(r=0.25 \mathrm{~mm})$. 

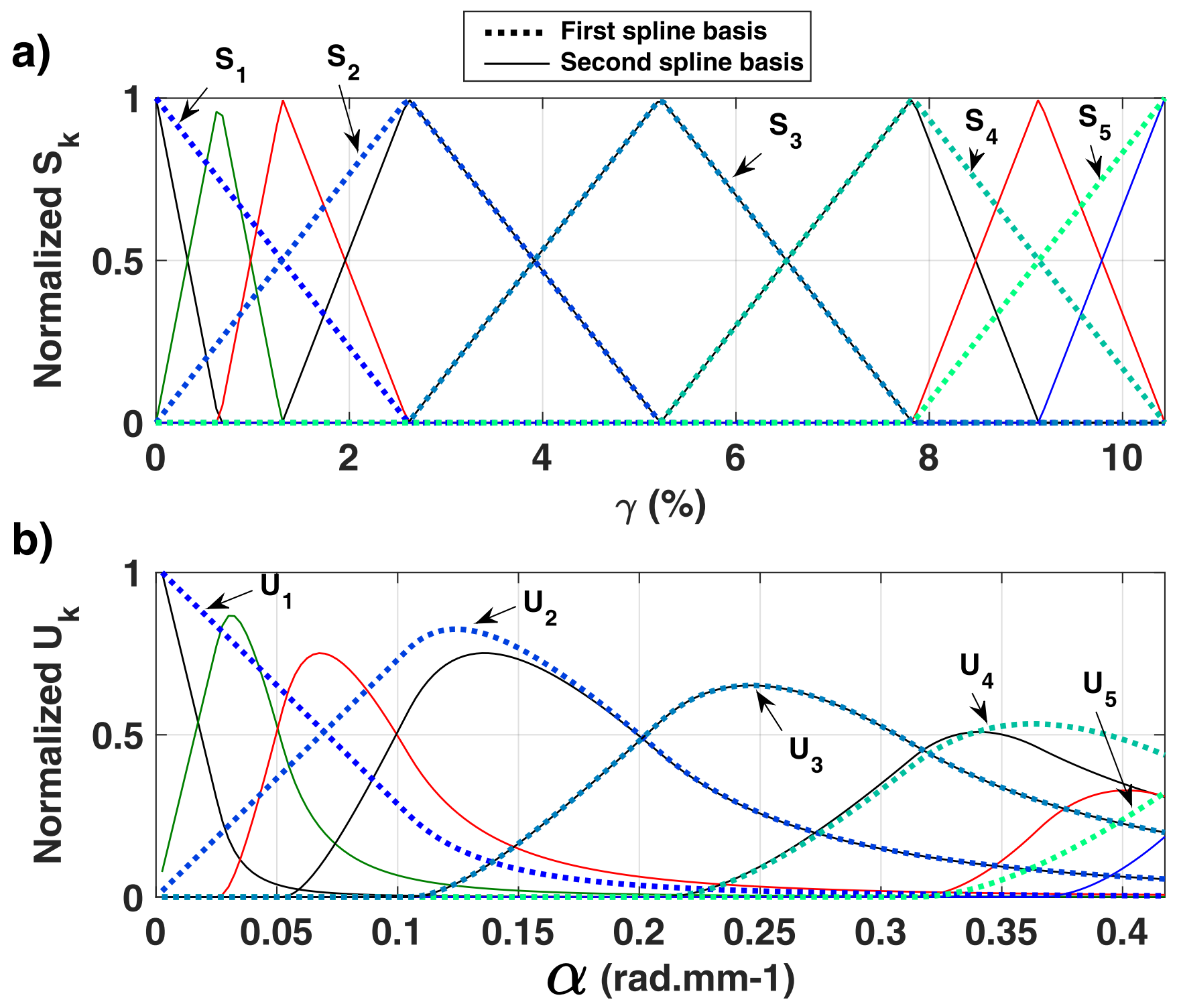

Figure 6: (a) Used linear splines basis: $\left\{S_{k}\right\} k \in[1,5]$ (dashed line) and associated refined basis $\left\{S_{k}\right\} k \in[1,8]$ (continuous line), (b) Associated normalized $U_{k}$ functions for each linear splines basis. 
a)
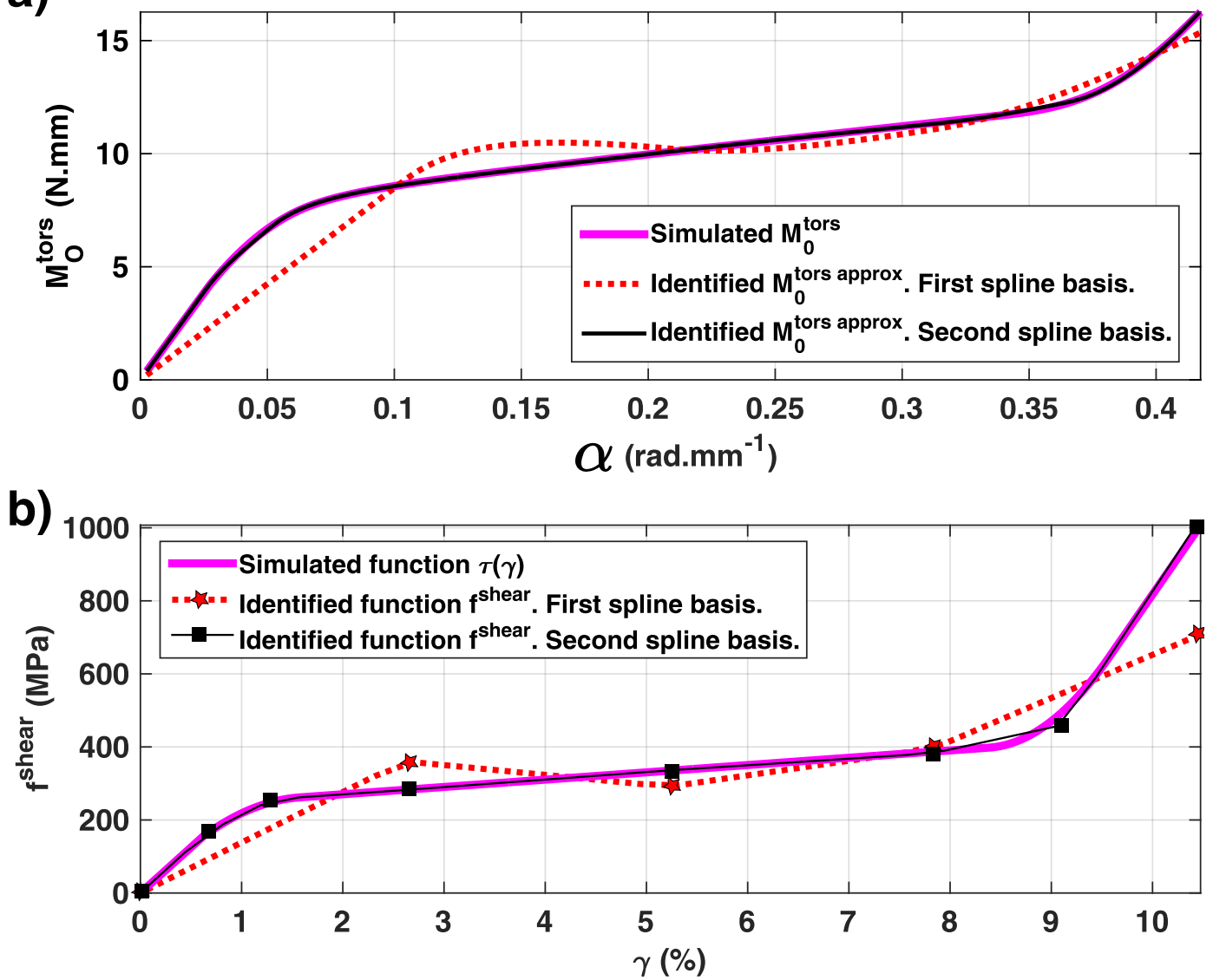

Figure 7: Identified results using two different linear splines basis $S_{k}$ : (a) Identified moment $\left\{M_{0}^{\text {tors approx }}\left(\alpha_{p}\right)\right\}$, (b) Identified function $f^{\text {shearapprox }}$ 

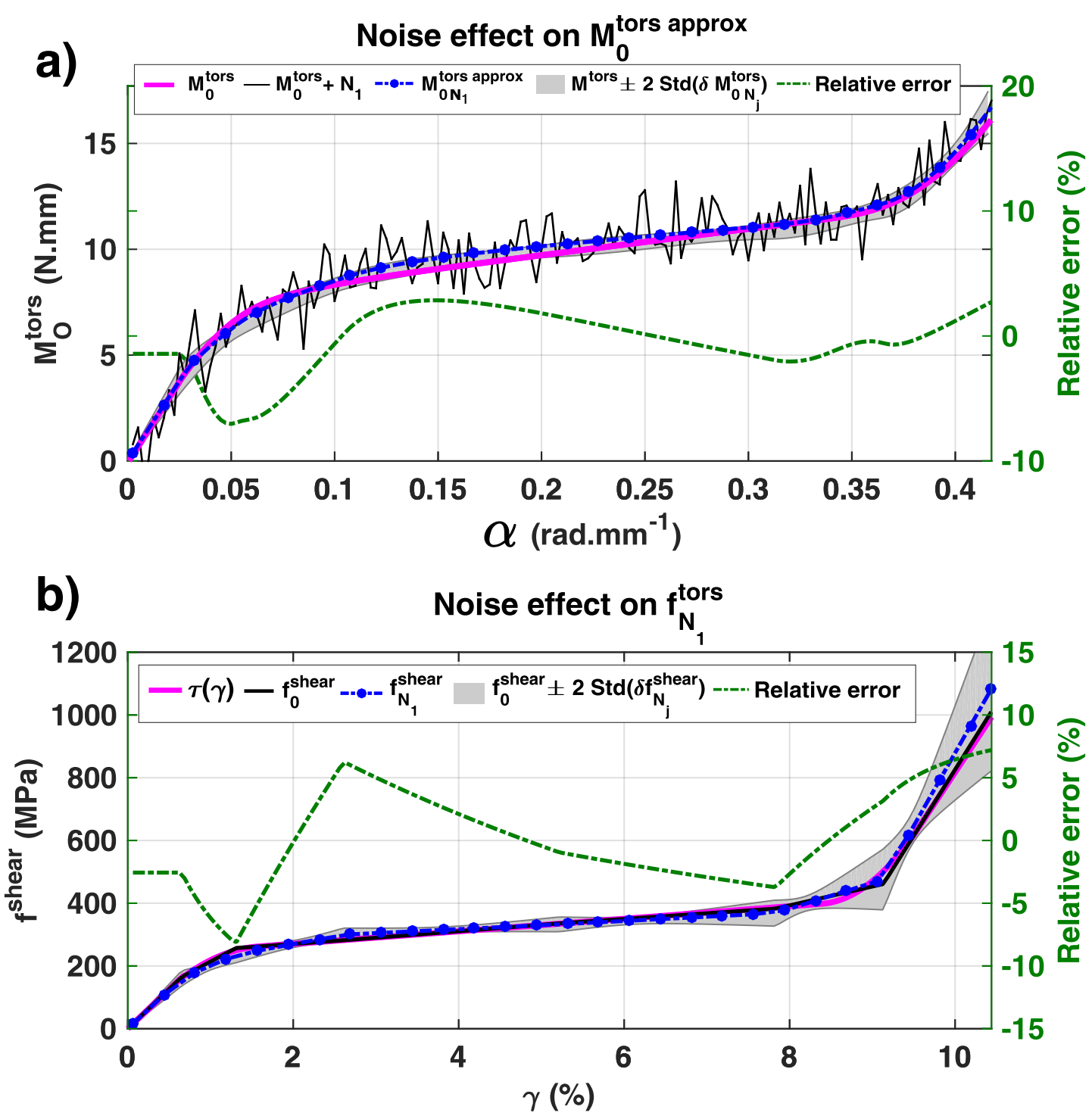

Figure 8: (a) Noiseless and noisy input signal $\left\{M_{0}^{\text {tors }}\left(C_{p}\right)\right\}$. Associated approximation $\left\{M_{0}^{\text {tors approx }}\left(\alpha_{p}\right)\right\}$ and errorbar associated to this noise level (left scale) and identification relative error (right scale), (b) Reference function $\tau(\gamma)$ and corresponding identified functions in noisy and noiseless conditions $f_{0}^{\text {shear }}$ and $f_{N_{1}}^{\text {shear }}$ (left scale). Relative error between solutions identified in noisy and noiseless conditions (right scale). 


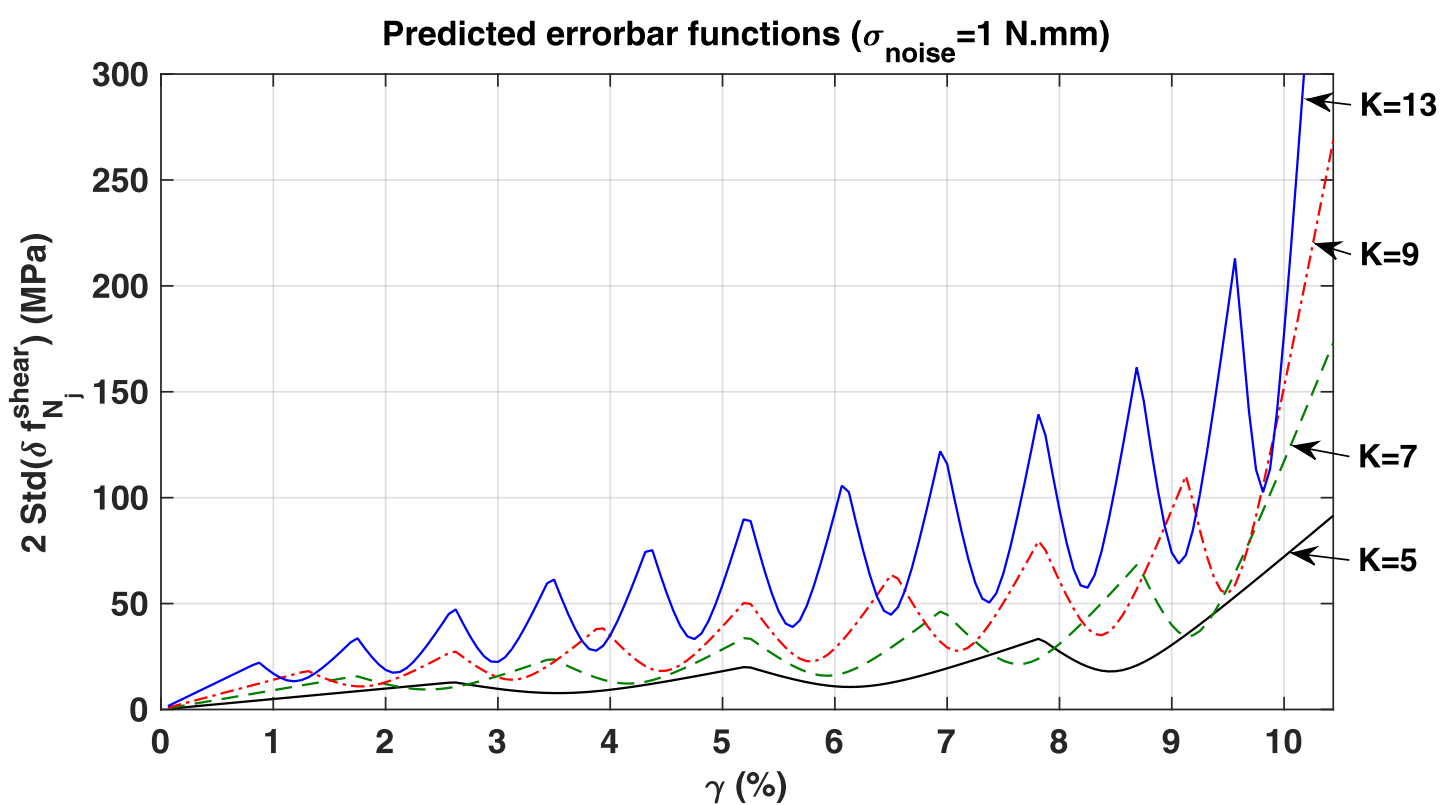

Figure 9: Error-bar functions $2 \operatorname{Std}\left(\delta f_{N_{j}}^{\text {shear }}\right)$ computed for different base spline families $K \in[5,7,9,13]$ and calculated for noise standard deviation $\sigma_{\text {noise }}=1 \mathrm{Nmm}$. 
a)

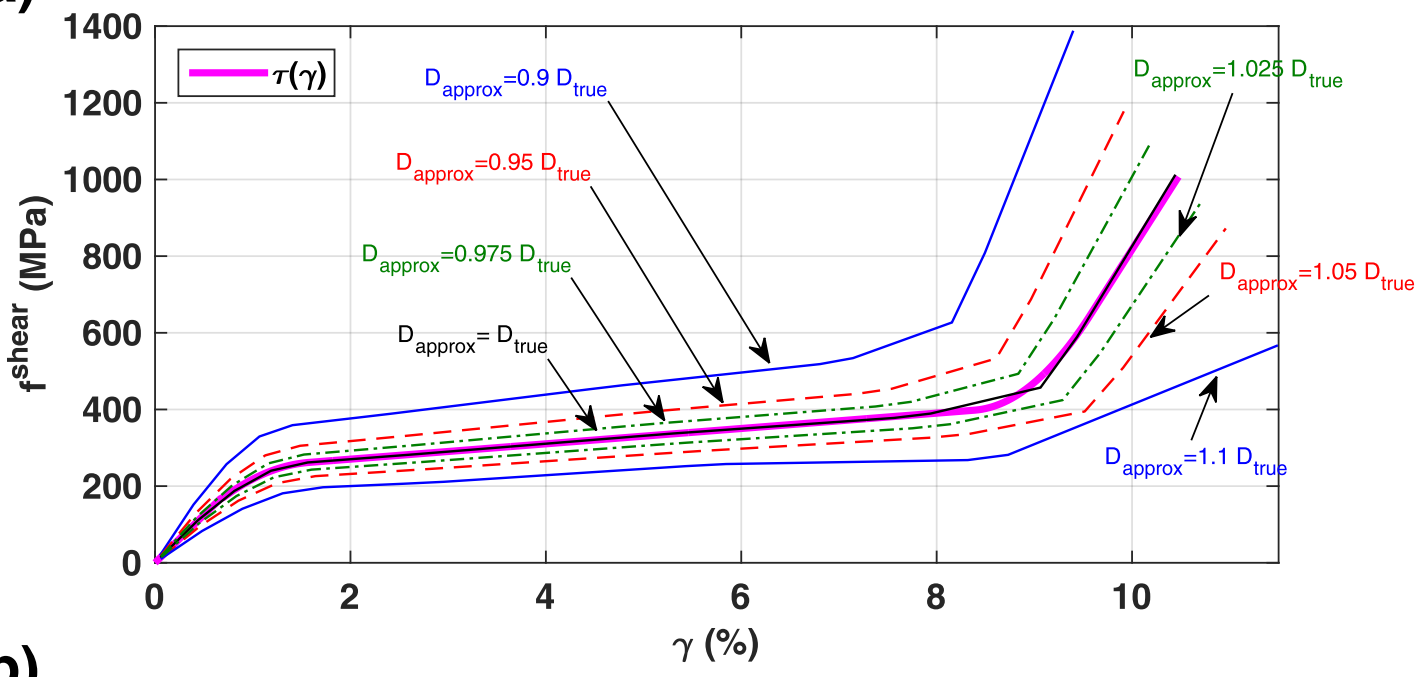

b)

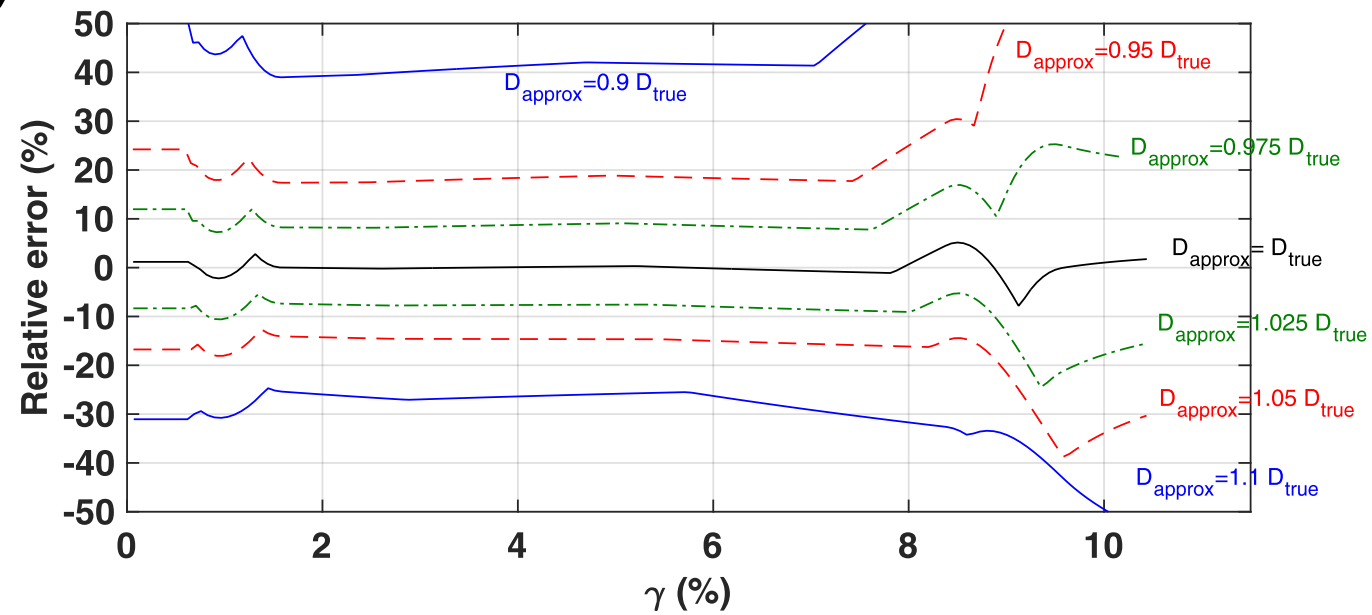

Figure 10: Identification errors induced by error on the specimen diameter: a) Identified shear function $f^{\text {shear }}$, b) Identification relative errors between the identified functions $f^{\text {shear }}$ and the input shear function $\tau(\gamma)$. 

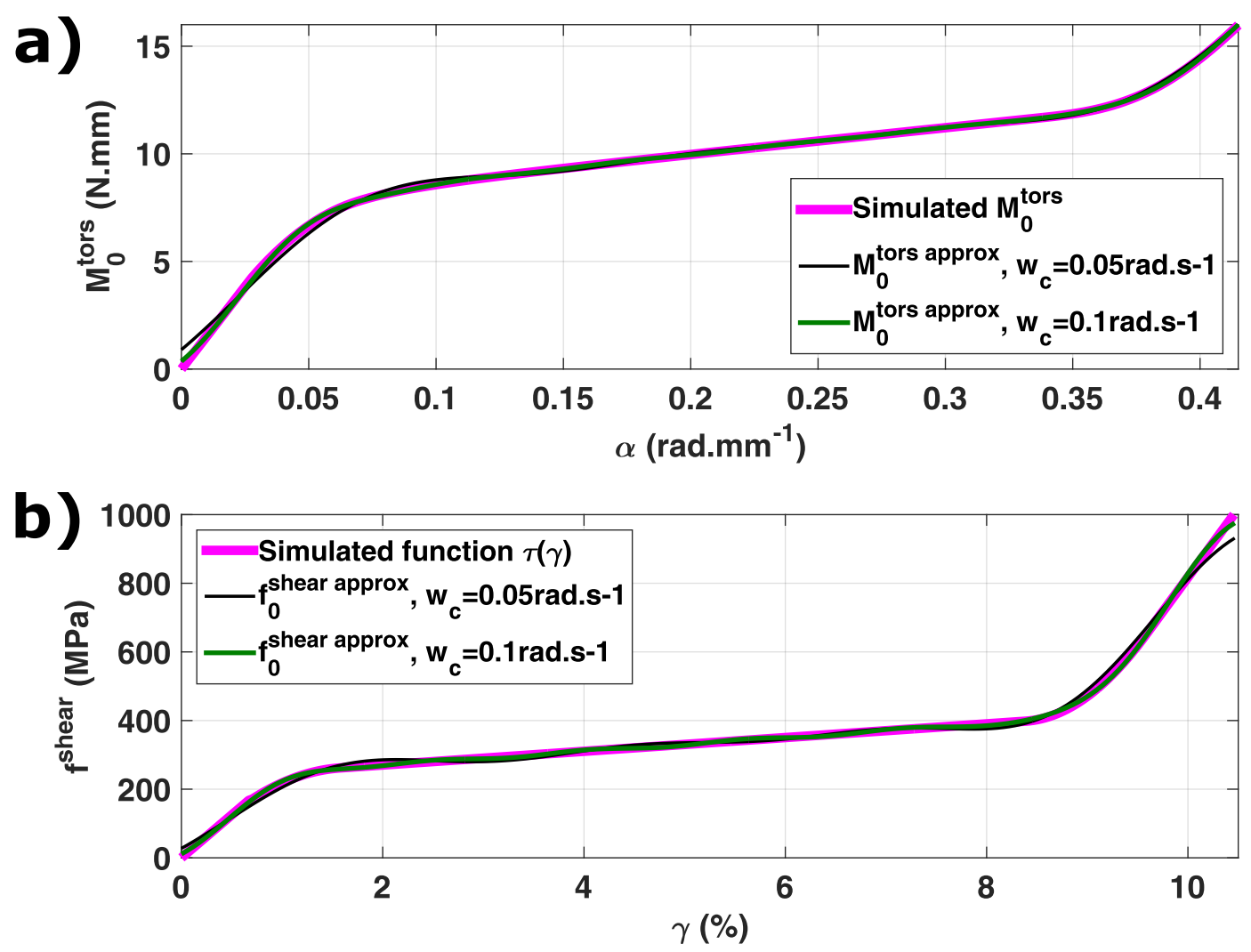

Figure 11: Identified results using the method presented in [11]: filtering using cut-off pulsations $w_{c}=0.05$ and $0.15 \mathrm{rad} \mathrm{s}^{-1}$ (a) Identified moment $\left\{M_{0}^{\text {tors approx }}\left(\alpha_{p}\right)\right\}$, (b) Identified function $f^{\text {shear approx }}$.

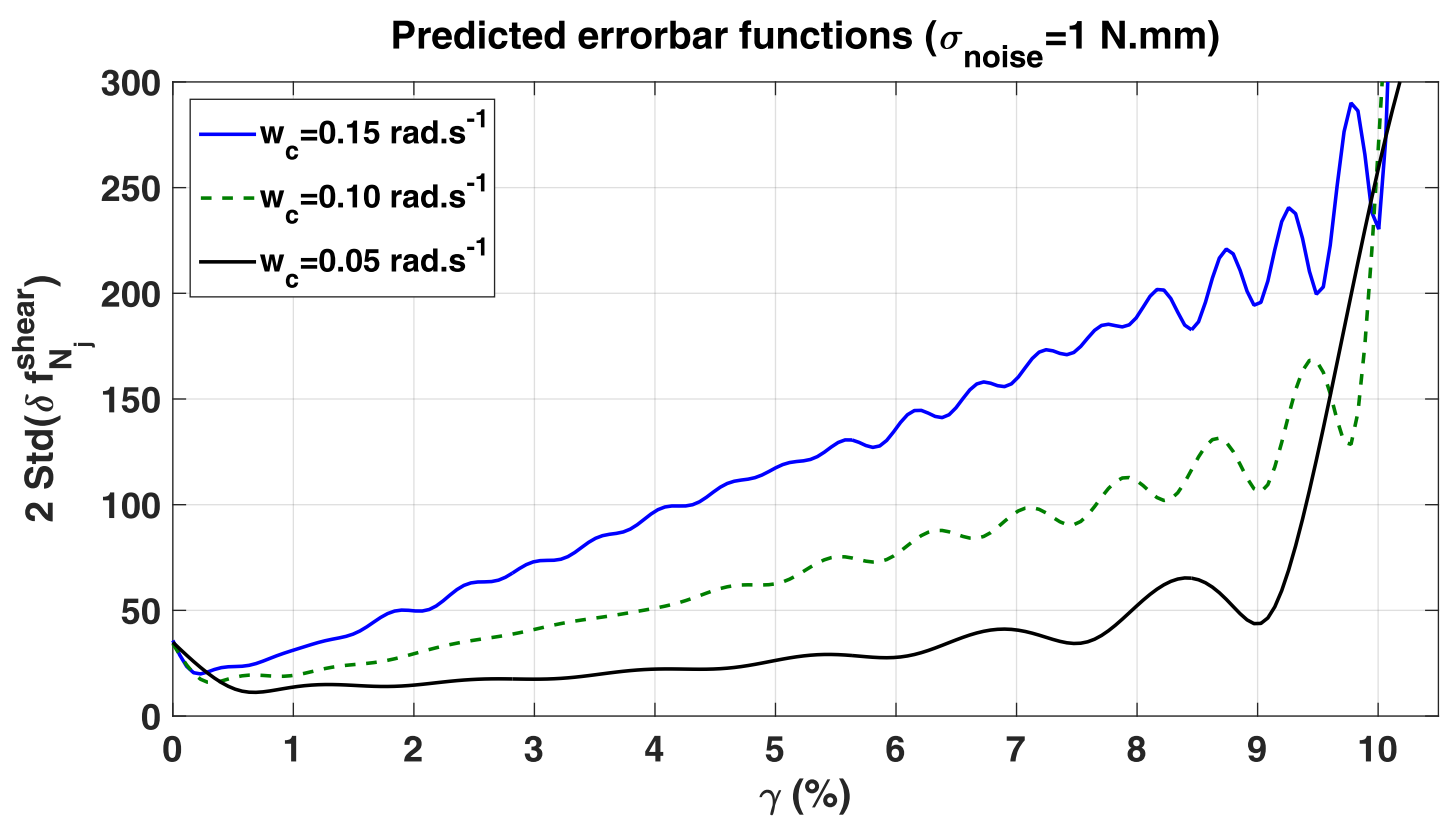

Figure 12: Error-bar functions $2 \operatorname{Std}\left(\delta f_{N_{j}}^{\text {shear }}\right)$ computed using the method presented in [11] for different cut-off pulsation $w_{c}$ for a noise standard deviation $\sigma_{n o i s e}=1 \mathrm{Nmm}$. 
a)

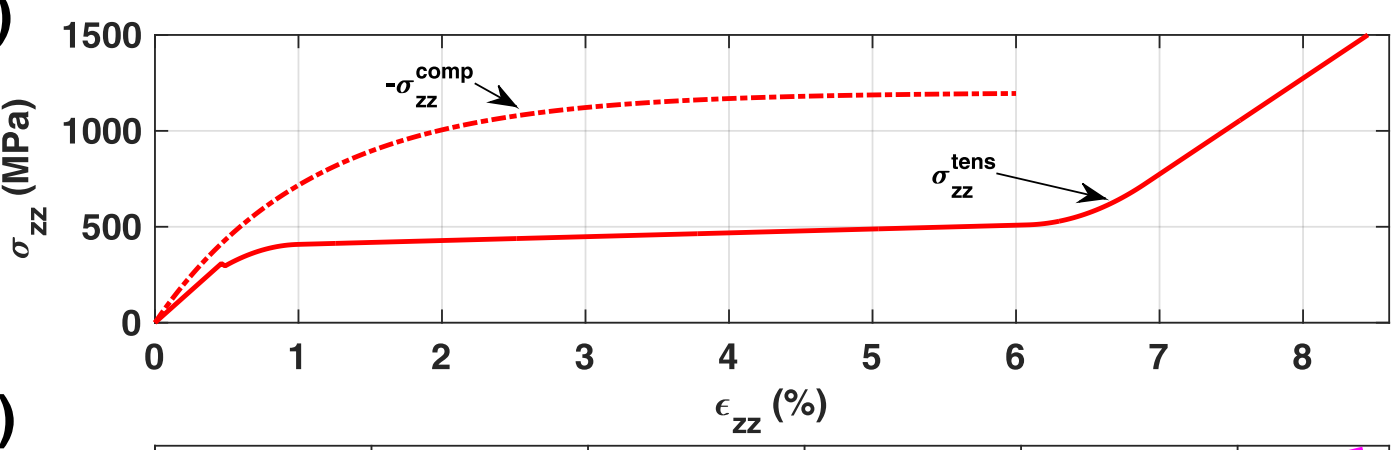

b)

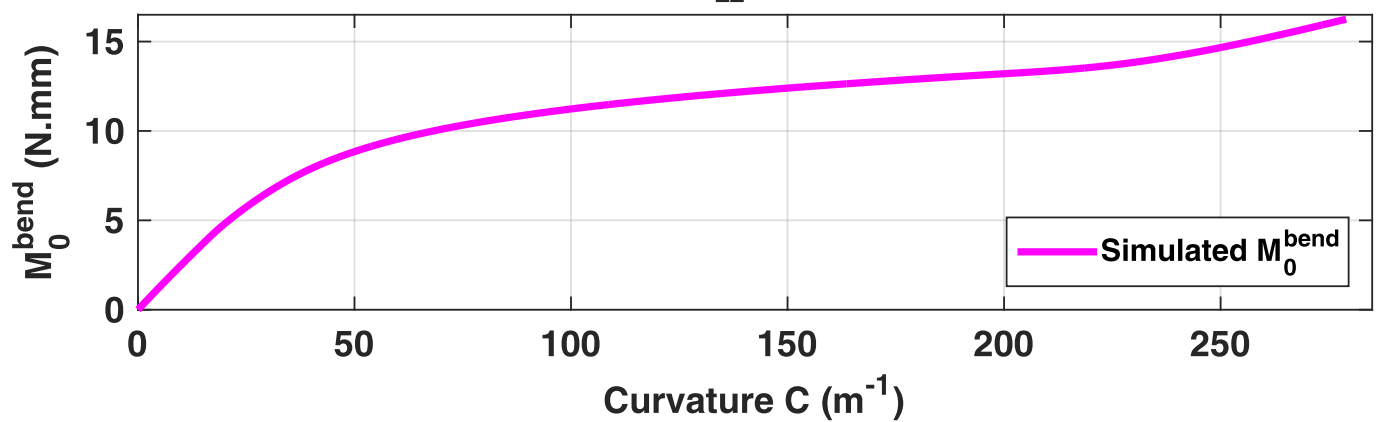

c)

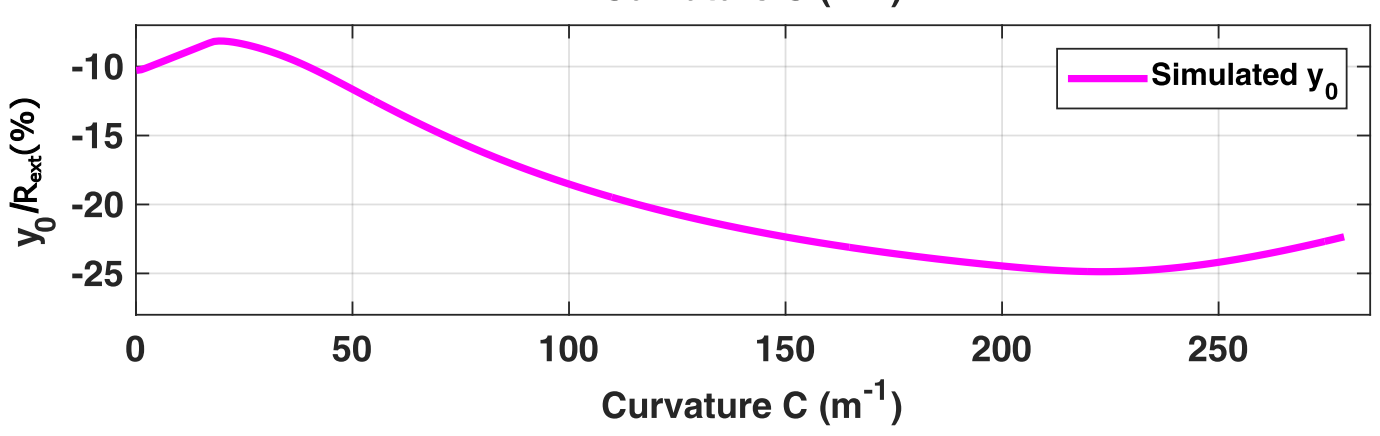

Figure 13: (a) Reference stress-strain function under uni-axial asymmetrical compressive and tensile behaviors (b) corresponding simulated pure bending moment $\left\{M_{0}^{\text {bend }}\left(C_{p}\right)\right\}$, (c) corresponding neutral surface relative position $\left\{y_{0}\left(C_{p}\right)\right\} / R_{\text {ext }}$. 


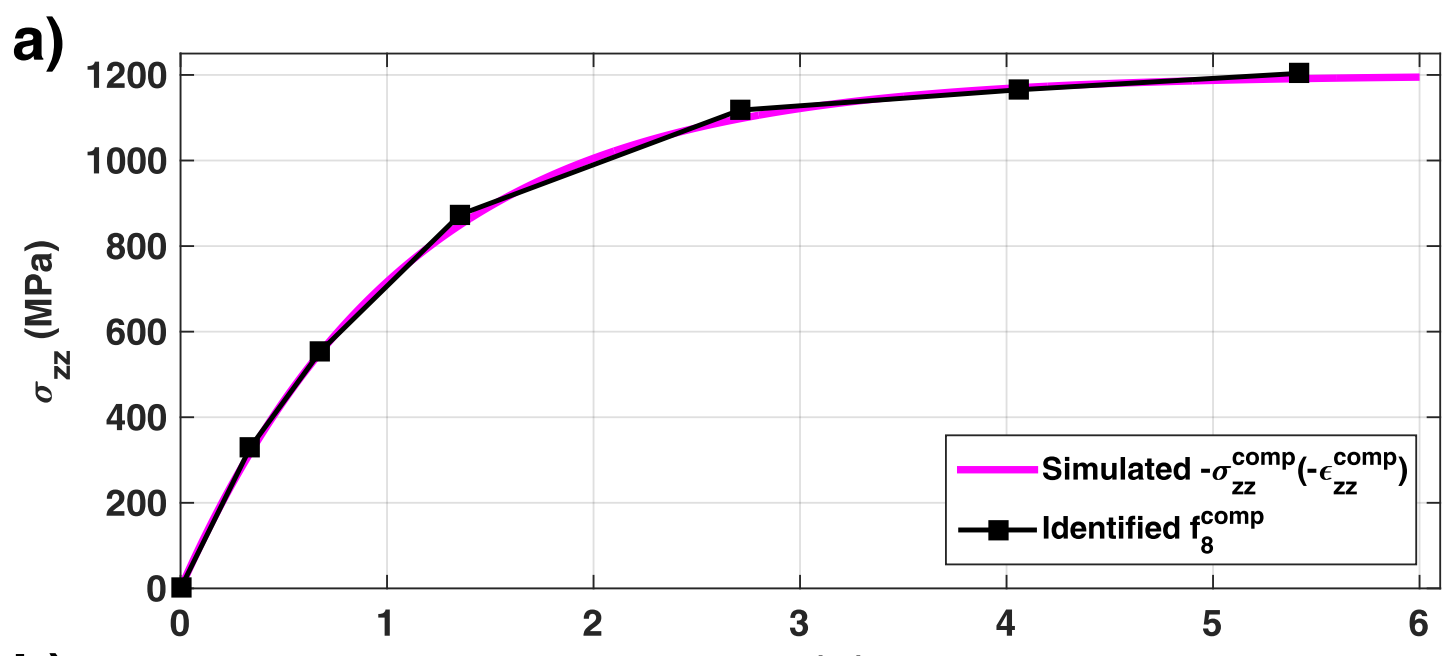

b)

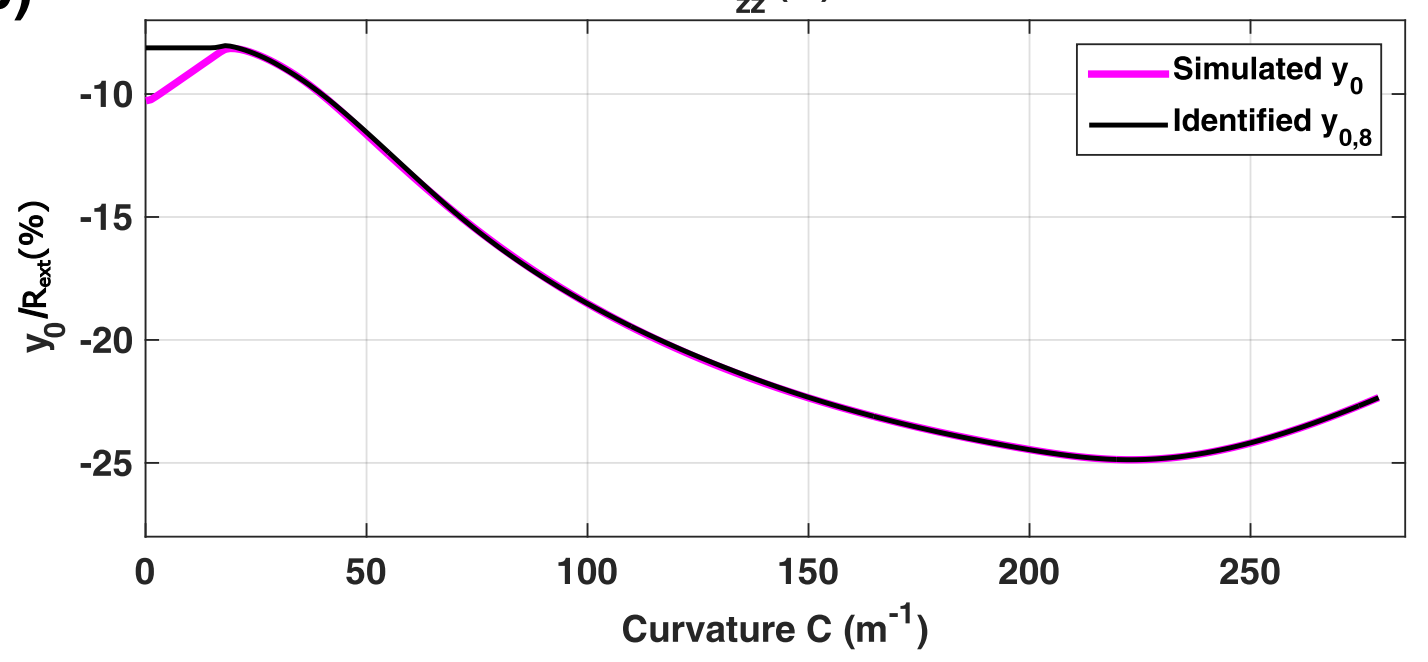

Figure 14: a) Identified stress-strain compression functions $f^{c o m p}$, b) Identified neutral surface relative position $\left\{y_{0}\left(C_{p}\right)\right\} / R_{e x t}$. 

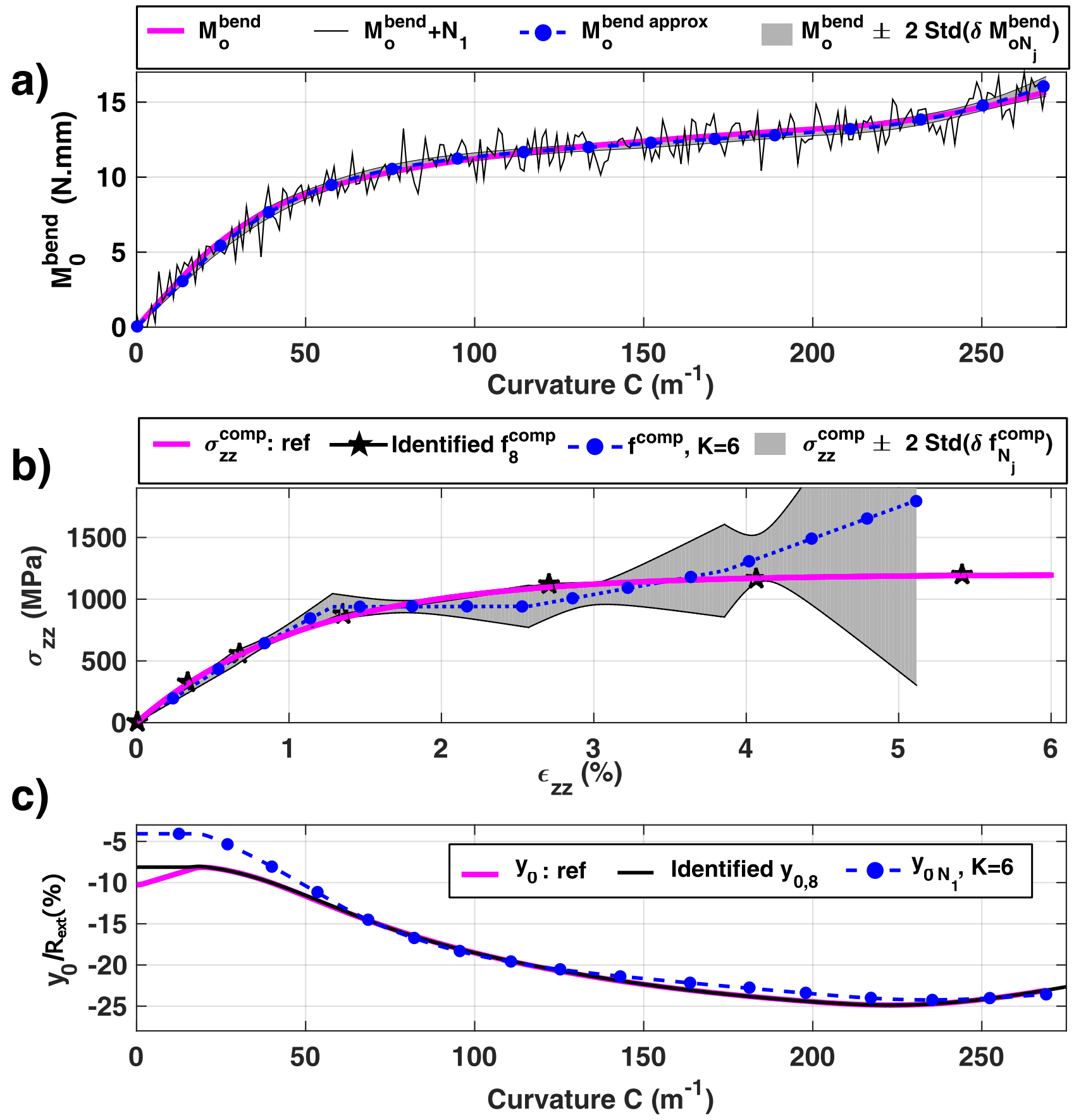

Figure 15: Identification robustness to noise on noisy bending moment: (a) Noiseless and noisy input signal $\left(\left\{M_{0}^{\text {bend }}\left(C_{p}\right)\right\}\right.$ and $\left\{M_{0}^{\text {bend }}\right\}+\left\{N_{1}\right\}$, respectively), identified bending moment $\left\{M_{0}^{\text {bend approx }}\left(C_{p}\right)\right\}$ and error-bar associated to this noise level, (b) Reference compression function $\sigma_{z z}^{\operatorname{com} p}$, corresponding identified function identified in noiseless and noisy conditions $\left(f_{0}^{c o m p}\right.$ and $f_{N_{1}}^{c o m p}$, respectively) and error-bar associated to this noise level, (c) Reference neutral surface relative position $\left\{y_{0}\left(C_{p}\right)\right\} / R_{e x t}$ and corresponding identified functions in noisy and noiseless conditions. 


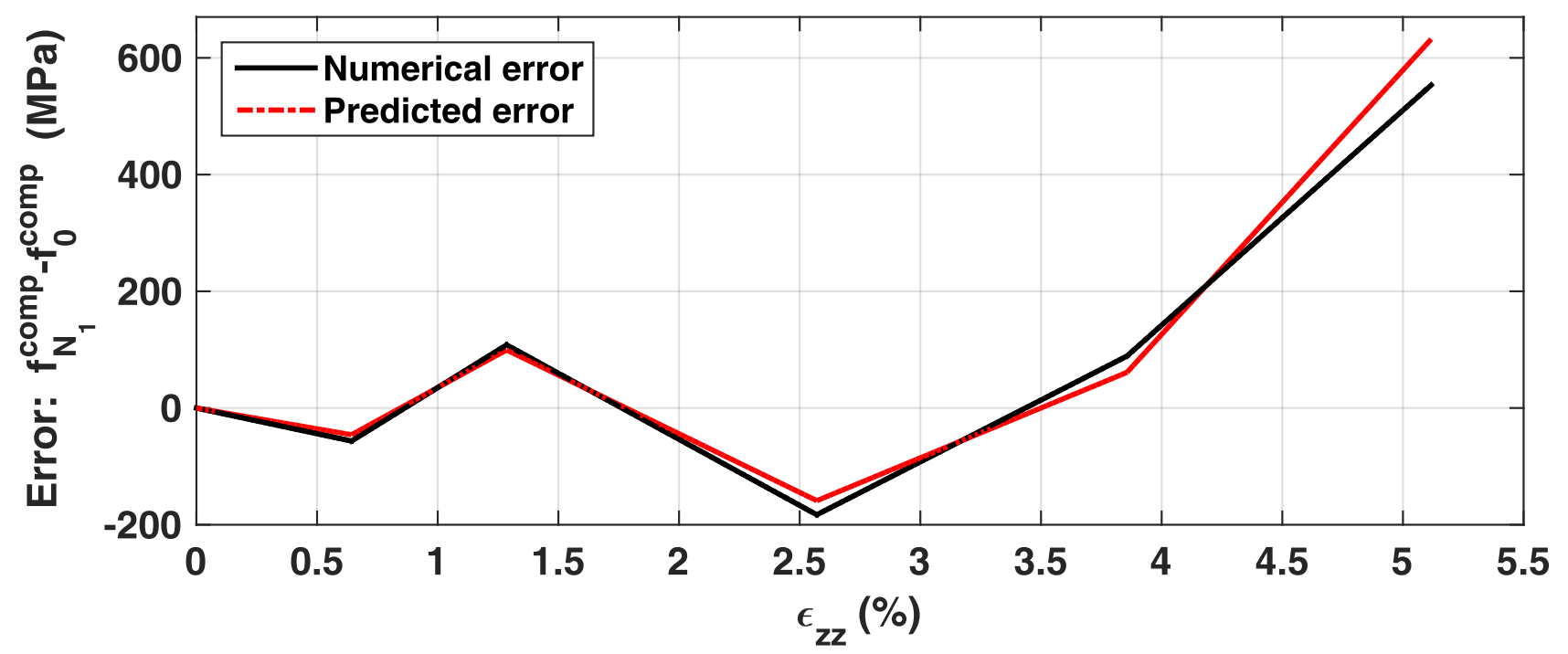

Figure 16: Comparison between predicted and numerical error on the identified function $f_{N_{1}}^{c o m p}$ induced by the noise copy $\left\{N_{1}\right\}$.
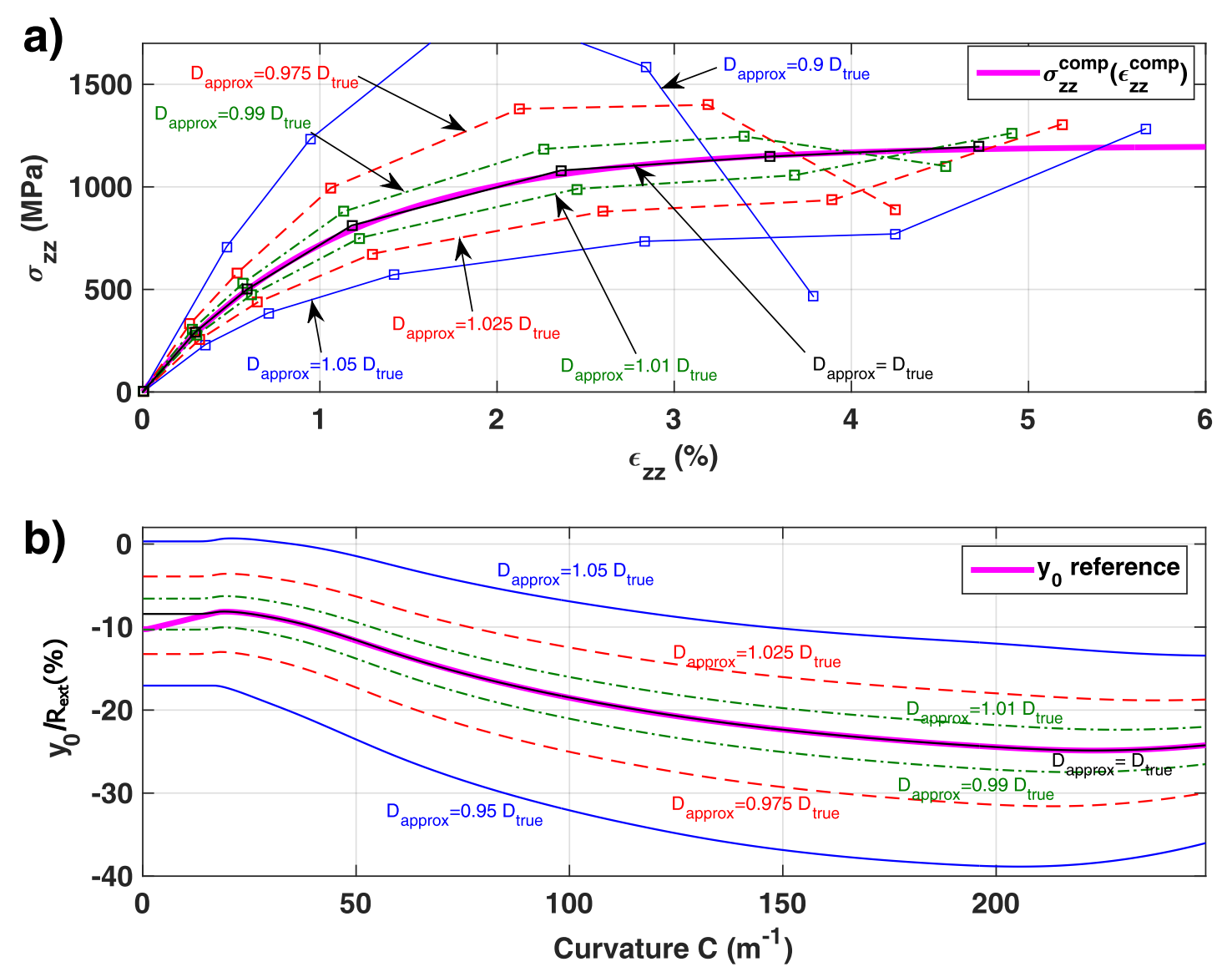

Figure 17: Error induced by error on the specimen diameter: a) Identified law $f^{\text {comp }}$, b) Identified neutral surface relative position $\left\{y_{0}\left(C_{p}\right)\right\} / R_{e x t}$. 